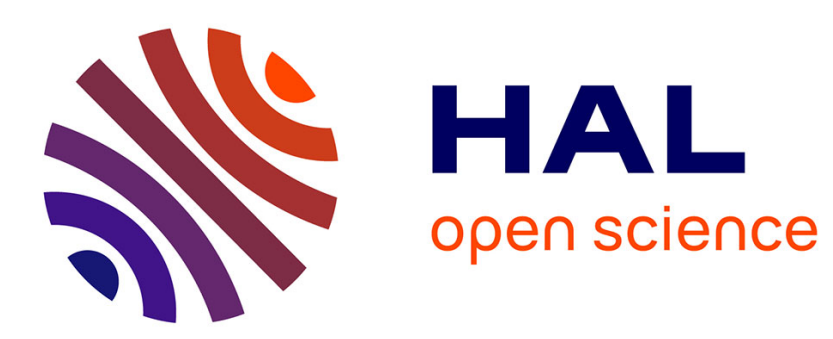

\title{
Minimizing the Stretch When Scheduling Flows of Divisible Requests
}

\author{
Arnaud Legrand, Alan Su, Frédéric Vivien
}

\section{To cite this version:}

Arnaud Legrand, Alan Su, Frédéric Vivien. Minimizing the Stretch When Scheduling Flows of Divisible Requests. Journal of Scheduling, 2008, 10.1007/s10951-008-0078-4 . hal-00789419

HAL Id: hal-00789419

https://hal.inria.fr/hal-00789419

Submitted on 16 Oct 2019

HAL is a multi-disciplinary open access archive for the deposit and dissemination of scientific research documents, whether they are published or not. The documents may come from teaching and research institutions in France or abroad, or from public or private research centers.
L'archive ouverte pluridisciplinaire HAL, est destinée au dépôt et à la diffusion de documents scientifiques de niveau recherche, publiés ou non, émanant des établissements d'enseignement et de recherche français ou étrangers, des laboratoires publics ou privés. 


\title{
Minimizing the stretch when scheduling flows of divisible requests
}

\author{
Arnaud Legrand ${ }^{1,4,6} \quad$ Alan $^{\mathrm{Su}^{2}} \quad$ Frédéric Vivien ${ }^{3,5,7}$ \\ ${ }^{1}$ CNRS ${ }^{2}$ Google Inc. ${ }^{3}$ INRIA ${ }^{4}$ Université de Grenoble ${ }^{5}$ Université de Lyon \\ ${ }^{6}$ LIG, UMR 5217, CNRS - Grenoble INP - INRIA - UJF - UPMF \\ 7 LIP, UMR 5668, ENS-Lyon - CNRS - INRIA - UCBL
}

\begin{abstract}
In this paper, we consider the problem of scheduling distributed biological sequence comparison applications. This problem lies in the divisible load framework with negligible communication costs. Thus far, very few results have been proposed for this model. We discuss and select relevant metrics for this framework: namely max-stretch and sum-stretch. We explain the relationship between our model and the preemptive single processor case, and we show how to extend algorithms that have been proposed in the literature for the single processor model to the divisible multi-processor problem domain. We recall known results on closely related problems, we show how to minimize the max-stretch on unrelated machines either in the divisible load model or with preemption, we derive new lower bounds on the competitive ratio of any online algorithm, we present new competitiveness results for existing algorithms, and we develop several new online heuristics. We also address the Pareto optimization of max-stretch. Then, we extensively study the performance of these algorithms and heuristics under realistic scenarios. Our study shows that all previously proposed guaranteed heuristics for max-stretch for the single processor model are inefficient in practice. In contrast, we show that our online algorithms based on linear programming are in practice near-optimal solutions for max-stretch. Our study also clearly suggests heuristics that are efficient for both metrics, although a combined optimization is in theory not possible in the general case.
\end{abstract}

\section{Introduction}

The problem of searching large-scale genomic and proteomic sequence databanks is an increasingly important bioinformatics problem. The results we present in this paper concern the deployment of such applications in heterogeneous parallel computing environments. In the genomic sequence comparison scenario, the presence of the required databank on a particular node is the sole factor that constrains task placement decisions. This application is thus part of a larger class of applications, in which each task in the application workload exhibits an "affinity" for particular nodes of the targeted computational platform. In this context, task affinities are determined by location and replication of the sequence databanks in the distributed platform.

Numerous efforts to parallelize biological sequence comparison applications have been realized (e.g., [13, $15,32]$ ). These efforts are facilitated by the fact that such biological sequence comparison algorithms are typically computationally intensive, embarrassingly parallel workloads. In the scheduling literature, this computational model is effectively a divisible workload scheduling problem $[9,11]$ with negligible communication overheads. The work presented in this paper concerns this application model, particularly in the context of online scheduling (i.e., in which the scheduler has no knowledge of any job in the workload in advance of its release date). Thus far, this specific problem has not been considered in the scheduling literature.

Aside from divisibility, the main difference with classical scheduling problems lies in the fact that the platforms we target are shared by many users. Consequently, we need to ensure a certain degree of fairness between the different users and requests. Defining a fair objective that accounts for the various job characteristics (release date, processing time) is thus the first difficulty to overcome. After 
having presented our motivating application and our framework in Section 2, we review various classical metrics in Section 3 and conclude that the stretch of a job is an appropriate basis for evaluation. As a consequence, we mainly focus on the max-stretch and sum-stretch metrics. To have a good background on related objectives functions and results, in Section 4 we focus on the max-flow and sum-flow metrics. Then in Section 5 we study sum-stretch optimization, in Section 6 offline max-stretch optimization, and in Section 7 Pareto offline optimization of max-stretch. Building on the previous sections, we focus in Section 8 on the online optimization of max-stretch. This paper contains no section devoted to the related work as the related work will be discussed throughout this article. However, we summarize in the conclusion the known and new results on complexity. Finally, we present in Section 9 an experimental evaluation of the different solutions proposed, and we conclude in Section 10.

The main contributions of this work are:

- OfFLINE SUM-FLOW AND SUM-STRETCH. We show that sum-flow minimization is NP-complete on unrelated machines under the divisible load model $\left(\left\langle R\left|r_{j}, \operatorname{div}\right| \sum F_{j}\right\rangle\right.$ is NP-complete). We also show that sum-stretch minimization is NP-complete on one machine without preemption and also on unrelated machines under the divisible load model $\left(\left\langle 1\left|r_{j}\right| \sum S_{j}\right\rangle\right.$ and $\left\langle R\left|r_{j}, d i v\right| \sum S_{j}\right\rangle$ are NPcomplete).

- OfFLINE MAX WEIGHTED FLOW. We present polynomial-time algorithms to solve the minimization of max weighted flow, offline, on unrelated machines, in the divisible load model and in the preemptive model: $\left\langle R\left|r_{j} ; d i v\right| \max w_{j} F_{j}\right\rangle$ and $\left\langle R\left|r_{j} ; p m t n\right| \max w_{j} F_{j}\right\rangle$ are polynomial.

We also propose heuristics to solve the offline Pareto minimization of max weighted flow, either on one machine or on unrelated machines. We present some cases in which these heuristics are optimal and we prove that the offline Pareto minimization of max-flow on unrelated machines is NP-complete.

- ONLINE SUM-STRETCH AND MAX-STRETCH. We show that First come, first served (FCFS) is $\Delta^{2}$ competitive for sum-stretch minimization and $\Delta$-competitive for max-stretch, where $\Delta$ denotes the ratio of the sizes of the largest and shortest jobs submitted to the system. We also prove that no online algorithm has simultaneously better competitive ratios for these two metrics.

We show that no online algorithm has a competitive ratio less than or equal to 1.19484 for the minimization of sum-stretch, or less than or equal to $\frac{1}{2} \Delta^{\sqrt{2}-1}$ for the minimization of max-stretch. (The previous known bounds were respectively 1.036 and $\frac{1}{2} \Delta^{\frac{1}{3}}$.)

For minimizing the sum-stretch on one machine with preemption, we show that Smith's ratio rule - which is then equivalent to shortest processing time - is not a competitive algorithm and that shortest weighted remaining processing time is at best 2-competitive.

Finally, we propose new heuristics for the online optimization of max-stretch. Through extensive simulations we compare them with solutions found in the literature and we show their very good performance.

All the details and proofs missing from this article can be found in its companion research report [28].

\section{Motivating Application and Framework}

\subsection{Motivating Application}

The only purpose of this section is to present the application that originally motivated this work, the GriPPS [10, 20] protein comparison application. The GriPPS framework is based on large databases of information about proteins; each protein is represented by a string of characters denoting the sequence of amino acids of which it is composed. Biologists need to search such sequence databases for specific patterns that indicate biologically significant structures. The GriPPS software enables such queries in grid environments, where the data may be replicated across a distributed heterogeneous computing platform.

As a matter of fact, there seems to be two common usages in protein comparison applications. In the first case, a biologist working on a set of proteins builds a pattern to search for similar sequences on the 
servers (this is the case for the GriPPS framework). In the second case, canonical patterns are known and should be used for comparison with daily updates of the databanks. This is the only case we are aware of where a very large set of motifs is sent to all databanks. This is however a typical background process whereas the first case is a typical online problem as many biologists concurrently use the servers. Therefore in this first case, the motifs are very small and communication cost they incur can really be neglected. To develop a suitable application model for the GriPPS application scenario, we performed a series of experiments to analyze the fundamental properties of the sequence comparison algorithms used in this code. Here we report on the conclusions of this study whose details can be found in Legrand, Su and Vivien $[26,25]$.

From our modeling perspective, the critical components of the GriPPS application are:

1. protein databanks: the reference databases of amino acid sequences, located at fixed locations in a distributed heterogeneous computing platform.

2. motifs: compact representations of amino acid patterns that are biologically important and serve as user input to the application.

3. sequence comparison servers: computational processes co-located with protein databanks that accept as input sets of motifs and return as output all matching entries in any subset of a particular databank.

The main characteristics of the GriPPS application are:

1. negligible communication costs. A motif is a relatively compact representation of an amino acid pattern. Therefore, the communication overhead induced while sending a motif to any processor is negligible compared to the processing time of a comparison.

2. divisible loads. The processing time required for sequence comparisons against a subset of a particular databank is linearly proportional to the size of the subset. This property allows us to distribute the processing of a request among many processors at the same time without additional cost.

The GriPPS protein databank search application is therefore an example of a linear divisible workload without communication costs.

In the classical scheduling literature, preemption is defined as the ability to suspend a job at any time and to resume it, possibly on another processor, at no cost. Our application implicitly falls in this category. Indeed, we can easily halt the processing of a request on a given processor and continue the pattern matching for the unprocessed part of the database on a different processor (as it only requires a negligible data transfer operation to move the pattern to the new location). From a theoretical perspective, divisible load without communication costs can be seen as a generalization of the preemptive execution model that allows for simultaneous execution of different parts of a same job on different machines.

3. uniform machines with restricted availabilities. A set of jobs is uniform over a set of processors if the relative execution times of jobs over the set of processors does not depend on the nature of the jobs. More formally, for any job $J_{j}$, the time $p_{i, j}$ needed to process job $J_{j}$ on processor $i$ is equal to $W_{j} \cdot c_{i}$, where $c_{i}$ describes the speed of processor $i$ and $W_{j}$ represents the size of $J_{j}$. Our experiments indicated a clear constant relationship between the computation time observed for a particular motif on a given machine, compared to the computation time measured on a reference machine for that same motif. This trend supports the hypothesis of uniformity. However, in practice a given databank may not be available on all sequence comparison servers. Our model essentially represents a uniform machines with restricted availabilities scheduling problem, which is a specific instance of the more general unrelated machines scheduling problem.

\subsection{Framework and Notations}

Formally, an instance of our problem is defined by $n$ jobs, $J_{1}, \ldots, J_{n}$ and $m$ machines (or processors), $M_{1}, \ldots, M_{m}$. The job $J_{j}$ arrives in the system at time $r_{j}$ (expressed in seconds), which is its release date; we suppose that jobs are numbered by increasing release dates. 
The value $p_{i, j}$ denotes the amount of time it would take for machine $M_{i}$ to process job $J_{j}$. Note that $p_{i, j}$ can be infinite if the job $J_{j}$ cannot be executed on the machine $M_{i}$, e.g., for our motivating application, if job $J_{j}$ requires a databank that is not present on the machine $M_{i}$. Finally, each job is assigned a weight or priority $w_{j}$.

As we have seen, for the particular case of our motivating application, we could replace the unrelated times $p_{i, j}$ by the expression $W_{j} \cdot c_{i}$, where $W_{j}$ denotes the size (in Mflop) of the job $J_{j}$ and $c_{i}$ denotes the computational capacity of machine $M_{i}$ (in second $\cdot \mathrm{Mflop}^{-1}$ ). To maintain correctness for the biological sequence comparison application, we separately maintain a list of databanks present at each machine and enforce the constraint that a job $J_{j}$ may only be executed on a machine that has a copy of all data upon which job $J_{j}$ depends. However, since the theoretical results we present do not rely on these restrictions, we retain the more general scheduling problem formulation that is, we address the unrelated machines framework in this article. As a consequence, all the values we consider in this article are nonnegative rational numbers (except the previously mentioned case in which $p_{i, j}$ is infinite if $J_{j}$ cannot be processed on $\left.M_{i}\right)$.

The time at which job $J_{j}$ is completed is denoted as $C_{j}$. Then, the flow time of the job $J_{j}$, defined as $F_{j}=C_{j}-r_{j}$, is essentially the time the job spends in the system.

Due to the divisible load model, each job may be divided into an arbitrary number of sub-jobs, of any size. Furthermore, each sub-job may be executed on any machine at which the data dependences of the job are satisfied. Thus, at a given moment, many different machines may be processing the same job (with a master scheduler ensuring that these machines are working on different parts of the job). Therefore, if we denote by $\alpha_{i, j}$ the fraction of job $J_{j}$ processed on $M_{i}$, we enforce the following property to ensure each job is fully executed: $\forall j, \sum_{i} \alpha_{i, j}=1$.

When a size $W_{j}$ can be defined for each job $J_{j}$ - e.g., in the single processor case - we denote by $\Delta$ the ratio of the sizes of the largest and shortest jobs submitted to the system: $\Delta=\frac{\max _{j} W_{j}}{\min _{j} W_{j}}$.

\subsection{Relationships with the Single Processor Case with Preemption}

We first prove that any schedule in the uniform machines model with divisibility has a canonical corresponding schedule in the single processor model with preemption. This is especially important as many interesting results in the scheduling literature only hold for the preemptive computation model (denoted pmtn).

Lemma 1. For any platform $M_{1}, \ldots, M_{m}$ composed of uniform processors, i.e., such that for any job $J_{j}, p_{i, j}=W_{j} \cdot c_{i}$, one can define a platform made of a single processor $\widetilde{M}$ with $\widetilde{c}=1 / \sum_{i} \frac{1}{c_{i}}$, such that: For any divisible schedule of $J_{1}, \ldots, J_{n}$ on $\left\{M_{1}, \ldots, M_{m}\right\}$ there exists a preemptive schedule of $J_{1}, \ldots$, $J_{n}$ on $\widetilde{M}$ with smaller or equal completion times.

Conversely, for any preemptive schedule of $J_{1}, \ldots, J_{n}$ on $\widetilde{M}$ there exists a divisible schedule of $\left\{M_{1}, \ldots, M_{m}\right\}$ with equal completion times.

Figure 1 illustrates the underlying idea (see research report [28] for details). The reverse transformation simply processes jobs sequentially, distributing each job's work across all processors. As a consequence, any complexity result for the preemptive single processor model also holds for the uniform divisible model. Thus, throughout this article, in addition to addressing the multi-processor case, we will also closely examine the single processor case.

Unfortunately, this line of reasoning is no longer valid when the computational platform exhibits restricted availability, as defined in Section 2. In the single processor case, a schedule can be seen as a priority list of the jobs (see the article of Bender, Muthukrishnan, and Rajaraman [7] for example). For this reason, whenever we will present heuristics for the uniprocessor case they will follow the same basic approach: maintain a priority list of the jobs and at any moment, execute the one with the highest priority. In the multi-processor case with restricted availability, an additional scheduling dimension must be resolved: the spatial distribution of each job.

The example in Figure 2 explains the difficulty of this last problem. In the uniform situation, it is always beneficial to fully distribute work across all available resources: each job's completion time in situation $B$ is strictly better than the corresponding job's completion time in situation $A$. However, introducing restricted availability confounds this process. Consider a case in which tasks may be limited in their ability to utilize some subset of the platform's resources (e.g., their requisite data 


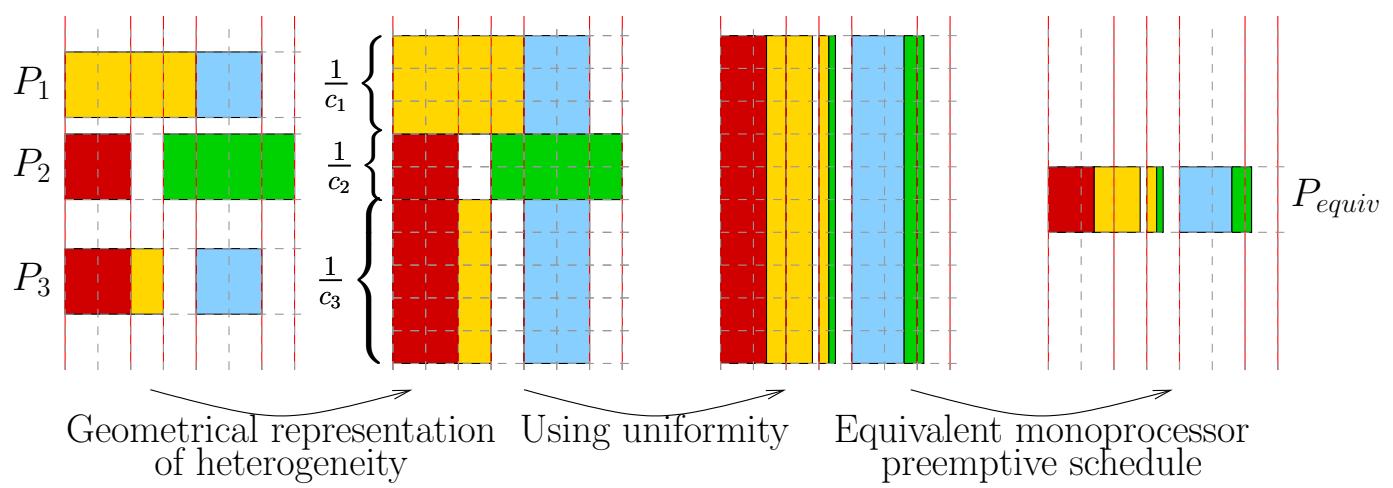

Figure 1: Geometrical transformation of a divisible uniform problem into a preemptive single processor problem

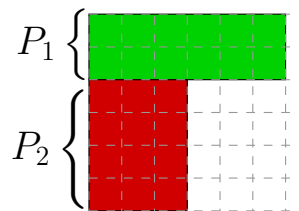

A: initial schedule

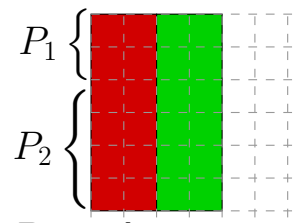

$B$ : uniform processing

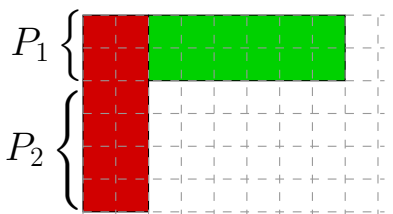

$C$ : restricted availability

Figure 2: Illustrating the difference between the uniform model and the restricted availability model.

are not present throughout the platform). In situation $C$ of Figure 2, one task is subject to restricted availability: the $P_{2}$ computational resource is not able to service this task. Deciding between various scheduling options in this scenario is non-trivial in the general case (for example schedule A has a better max flow than schedule $\mathrm{C}$, but schedule $\mathrm{C}$ has a better max stretch than schedule $\mathrm{A}$ ), so we apply the following simple rule to build a schedule for general platforms from single processor heuristics:

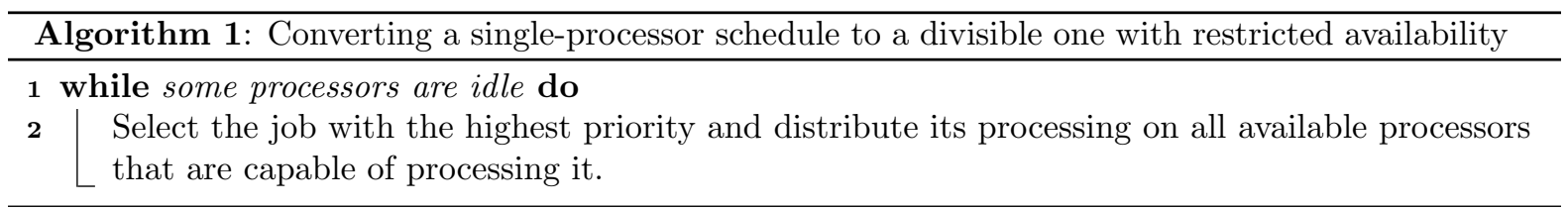

An other important characteristic of our problem is that we target a platform shared by many users. As a consequence, we need to ensure a certain degree of fairness between the different requests. Given a set of requests, how should we share resources amongst the different requests? The next section examines objective functions that are well-suited to achieve this notion of fairness.

\section{Objective Functions}

We first recall several common objective functions in the scheduling literature and highlight those that are most relevant to our work (Section 3.1). Then, we show that the optimization of certain objectives are mutually exclusive (Section 3.2).

\subsection{Looking for a Fair Objective Function}

The most common objective function in the parallel scheduling literature is the makespan: the maximum of the job termination times, or $\max _{j} C_{j}$. Makespan minimization is conceptually a system-centric approach, seeking to ensure efficient platform utilization. Makespan minimization is meaningful when there is only one user and when all jobs are submitted simultaneously. However, individual users sharing a system are typically more interested in job-centric metrics, such as job flow time (also called response time): the time an individual job spends in the system. Optimizing the average (or total) flow time, 
$\sum_{j} F_{j}$, suffers from the limitation that starvation is possible, i.e., some jobs may be delayed to an unbounded extent [5]. By contrast, minimization of the maximum flow time, $\max _{j} F_{j}$, does not suffer from this limitation, but it tends to favor long jobs to the detriment of short ones. To overcome this problem, one common approach [14] focuses on the weighted flow time, using job weights to offset the bias against short jobs. Sum weighted flow and maximum weighted flow metrics can then be analogously defined. Note however that the starvation problem identified for sum-flow minimization is inherent to all sum-based objectives, so the sum weighted flow suffers from the same weakness. The stretch is a particular case of weighted flow, in which a job's weight is inversely proportional to its size: $w_{j}=1 / W_{j}[5]$. On a single processor, the stretch of a job can be seen as the slowdown it experiences when the system is loaded. In a network context, the stretch can be seen as the inverse of the overall bandwidth allocated to a given transfer (i.e., the amount of data to transfer divided by the overall time needed to complete the transfer). However this kind of definition does not account for the affinity of some tasks with some particular machines (e.g., the scarcity of a particular database). That is why we think a slightly different definition should be used in an unrelated machines context. The stretch is originally defined to represent the slowdown a job experiences when the system is loaded. In the remaining of this article, we will thus define the stretch as a particular case of weighted flow, in which a job's weight is inversely proportional to its processing time when the system is empty: $w_{j}=\sum_{i} \frac{1}{p_{i, j}}$ in our divisible load model. This definition matches the previous one in a single processor context and is thus a reasonably fair measure of the level of service provided to an individual job. It is more relevant than the flow in a system with highly variable job sizes. Consequently, this article focuses mainly on the sum-stretch $\left(\sum S_{j}\right)$ and the max-stretch $\left(\max S_{j}\right)$ metrics.

\subsection{Simultaneous Online Optimization of Sum-Stretch and Max-Stretch is Impossible}

We prove that simultaneously optimizing the objectives we have defined earlier (sum-stretch and maxstretch) may be impossible in certain situations ${ }^{1}$. In this section, we only consider the single processor case.

Theorem 1. Consider any online algorithm which has a competitive ratio of $\rho(\Delta)$ for the sum-stretch. We assume that this competitive ratio is not trivial, i.e., that $\rho(\Delta)<\Delta^{2}$. Then, there exists for this algorithm a sequence of jobs that leads to starvation, and thus for which the obtained max-stretch is arbitrarily greater than the optimal max-stretch.

Note that the currently best known online algorithm for sum-stretch is 2-competitive (see Section 5.3). Using the exact same construction, we can show that for any online algorithm which has a non-trivial competitive ratio of $\rho(\Delta)<\Delta$ for the sum-flow, there exists a sequence of jobs leading to starvation and where the obtained max-flow is arbitrarily greater than the optimal one.

We must comment on our assumption about non-trivial competitive ratios. This comes from the fact that ignoring job sizes leads on a single processor to a $\Delta^{2}$-competitive online algorithm for sum-stretch and $\Delta$-competitive online algorithm for sum-flow:

\section{Theorem 2. FCFS is:}

- $\Delta^{2}$-competitive for the online minimization of sum-stretch,

- $\Delta$-competitive for the online minimization of max-stretch,

- $\Delta$-competitive for the online minimization of sum-flow, and

- optimal for the online minimization of max-flow (classical result, see Bender et al. [5] for example).

Proofs and details can be found in the research report corresponding to this article [28]. We now prove Theorem 1.

Proof. Let us consider the case of an online algorithm for the sum-stretch optimization problem that achieves a competitive ratio of $\rho(\Delta)<\Delta^{2}$. We arbitrarily take a value for $\Delta>1$. Then, there exists

\footnotetext{
${ }^{1}$ Note that the following two theorems have been incorrectly stated in [27].
} 
$\varepsilon>0$, such that $\rho(\Delta)<\Delta^{2}-\varepsilon$. Finally, let $\alpha$ be any integer such that $\frac{1+\alpha \Delta}{1+\frac{\alpha}{\alpha}}>\Delta^{2}-\frac{\varepsilon}{2}$ (note that this is the case for any value of $\alpha$ which is large enough).

At date 0 arrives $\alpha$ jobs, $J_{1}, \ldots, J_{\alpha}$, of size $\Delta$. Let $k$ be any integer. Then, at any time unit $t$, $0 \leqslant t \leqslant k-1$, arrives a job $J_{\alpha+t+1}$ of size 1 .

A possible schedule would be to process each of the $k$ jobs of size 1 at its release date, and to wait for the completion of the last of these jobs before processing the jobs $J_{1}, \ldots, J_{\alpha}$. The sum-stretch would then be $k \times 1+\frac{k+\Delta}{\Delta}+\ldots+\frac{k+\alpha \Delta}{\Delta}=\frac{\alpha(\alpha+1)}{2}+k\left(1+\frac{\alpha}{\Delta}\right)$ and the max-stretch would be $\alpha+\frac{k}{\Delta}$. Even if it is not optimal for neither one nor the other criteria, we can still use it as an upper-bound.

In fact, with our hypotheses, the online algorithm cannot complete the execution of all the jobs $J_{1}$, $\ldots, J_{\alpha}$ as long as there are jobs of size 1 arriving at each time unit. Otherwise, suppose that at some date $t_{1}$, jobs $J_{1}, \ldots, J_{\alpha}$ have all been completed. Then, a certain number $k_{1}$ of unit-size jobs were completed before time $t_{1}$. The scenario which minimizes the sum-stretch under these constraints is to schedule first the $k_{1}$ jobs $J_{\alpha+1}, \ldots, J_{\alpha+k_{1}}$ at their release dates, then to schedule $J_{1}, \ldots, J_{\alpha}$, and then the remaining $k-k_{1}$ jobs of size 1 . The sum-stretch of the actual schedule can therefore not be smaller than the sum-stretch of this schedule, which is equal to:

$$
k_{1} \times 1+\frac{k_{1}+\Delta}{\Delta}+\ldots+\frac{k_{1}+\alpha \Delta}{\Delta}+\left(k-k_{1}\right)(1+\alpha \Delta)=\left(\frac{\alpha(\alpha+1)}{2}+\frac{\alpha k_{1}}{\Delta}\right)+k_{1}+\left(k-k_{1}\right)(1+\alpha \Delta) .
$$

However, as, by hypothesis, we consider a $\rho(\Delta)$-competitive algorithm, the obtained schedule must at most be $\rho(\Delta)$ times the optimal schedule. This implies that:

$$
\begin{gathered}
\left(\frac{\alpha(\alpha+1)}{2}+\frac{\alpha k_{1}}{\Delta}\right)+k_{1}+\left(k-k_{1}\right)(1+\alpha \Delta) \leqslant \rho(\Delta)\left(\frac{\alpha(\alpha+1)}{2}+k\left(1+\frac{\alpha}{\Delta}\right)\right) \quad \Leftrightarrow \\
-\alpha \Delta k_{1}+\frac{\alpha(\alpha+1)}{2}(1-\rho(\Delta))+\frac{\alpha k_{1}}{\Delta} \leqslant k\left(\rho(\Delta)\left(1+\frac{\alpha}{\Delta}\right)-(1+\alpha \Delta)\right) .
\end{gathered}
$$

Once the approximation algorithm has completed the execution of the jobs $J_{1}, \ldots, J_{\alpha}$ we can keep sending unit-size jobs for $k$ to become as large as we wish. Therefore, for the inequality not to be violated, we must have $\rho(\Delta)\left(1+\frac{\alpha}{\Delta}\right)-(1+\alpha \Delta) \geqslant 0$. However, we have by hypothesis $\rho(\Delta)<\Delta^{2}-\varepsilon$. Therefore, we must have $\Delta^{2}-\varepsilon>\frac{1+\alpha \Delta}{1+\frac{\alpha}{\alpha}}$, which contradicts the definition of $\alpha$. Therefore, the only possible behavior for the approximation algorithm is to delay the execution of at least one of the jobs $J_{1}, \ldots, J_{\alpha}$ until after the end of the arrival of the unit-size jobs, whatever the number of these jobs. This leads to the starvation of at least one of these jobs. Furthermore, the ratio of the obtained max-stretch to the optimal one is $\frac{\alpha+\frac{k}{\Delta}}{1+\alpha \Delta}=\frac{\alpha \Delta+k}{\Delta(\alpha \Delta+a)}$, which may be arbitrarily large.

Intuitively, algorithms targeting max-based metrics ensure that no job is left behind. Such an algorithm is thus extremely "fair" in the sense that everybody's cost (in our context the weighted flow or the stretch of each job) is made as close to the other ones as possible. Sum-based metrics tend to optimize instead the utilization of the platform. The previous theorem establishes that these two objectives can be in opposition on particular instances. As a consequence, it should be noted that any algorithm optimizing a sum-based metric has the particularly undesirable property of potential starvation. This observation, combined with the fact that the stretch is more relevant than the flow in a system with highly variable job sizes, motivates max-stretch as the metric of choice in designing scheduling algorithms in the GriPPS setting.

\section{Flow Optimization}

On a single processor, the max-flow is optimized by FCFS (see Bender et al. [5] for example). Using the remarks of Section 2.3, we can thus easily derive an online optimal algorithm for $\langle Q| r_{j}$; div $\left|F_{\max }\right\rangle$. We will see in Section 6 that $\langle R| r_{j}$; div $\left|\max w_{j} F_{j}\right\rangle$ can be solved in polynomial time using linear programing techniques.

Regarding, sum-flow, it was proved by Baker [1], using exchange arguments, that SRPT (shortest remaining processing time first) is optimal for the $\left\langle 1\left|r_{j} ; p m t n\right| \sum C_{j}\right\rangle$ problem. It is thus also optimal for $\left\langle 1\left|r_{j} ; p m t n\right| \sum F_{j}\right\rangle$ and, using the remarks of Section 2.3 , we can easily derive an online optimal algorithm 
for $\langle Q| r_{j} ;$ div $\left|\sum F_{j}\right\rangle$. We will however see in this section that under the uniform machines with restricted availabilities model, this problem is much harder.

Many of the reduction we propose in this article rely on the following strongly NP-hard problem [18]:

Definition 1 (3-Dimensional Matching (3DM)). Given three sets $U=\left\{u_{1}, \ldots, u_{m}\right\}, V=\left\{v_{1}, \ldots, v_{m}\right\}$, and $W=\left\{w_{1}, \ldots, w_{m}\right\}$, and a subset $S \subset U \times V \times W$ of size $n \geqslant m$, does $S$ contain a perfect matching, that is, a set $S^{\prime} \subseteq S$ of cardinality $m$ that covers every element in $U \cup V \cup W$ ?

Theorem 3. The scheduling problem $\langle R| r_{j}$, div $\left|\sum F_{j}\right\rangle$ is NP-complete.

The reduction is made from 3-Dimensional Matching and uses the same idea as Sitters [35] used to prove the strong NP-hardness of $\left\langle R|p m t n| \sum C_{j}\right\rangle$. It should be noted that, in the reduction we use, the machines are uniform machines with restricted availabilities. We refer the reader to the extended version of this document [28] where the whole proof can be found.

\section{Sum-Stretch Optimization}

In this section, we give various results regarding sum-stretch optimization. In Section 5.1, we establish the complexity of this problem in our framework. In the remaining sections, we focus on the one processor setting and study the competitiveness of "classical" heuristics.

\subsection{Complexity of the Offline Problem}

In the general case, without preemption and divisibility, minimizing the sum-stretch is an NP-complete problem:

Theorem 4. The scheduling problem $\left\langle 1\left|r_{j}\right| \sum S_{j}\right\rangle$ is NP-complete.

This NP-completeness result is proved by reduction from of PARTition. A complete proof can be found in the research report corresponding to this article [28].

The complexity of the offline minimization of the sum-stretch with preemption is still an open problem. At the very least, this is a hint at the difficulty of this problem. In the framework with preemption, Bender, Muthukrishnan, and Rajaraman [7] present a Polynomial Time Approximation Scheme (PTAS) for minimizing the sum-stretch with preemption. Chekuri and Khanna [14] present an approximation scheme for the more general sum weighted flow minimization problem. As these approximation schemes cannot be extended to work in an online setting, we will not discuss them further.

Moving to the divisible load framework, we can easily say that the complexity of $\left\langle Q\left|r_{j} ; \operatorname{div}\right| \sum S_{j}\right\rangle$ is open (using the remarks of Section 2.3). The minimization of the sum-stretch is however NP-complete on unrelated machines:

Theorem 5. The scheduling problem $\langle R| r_{j}$, div $\left|\sum S_{j}\right\rangle$ is NP-complete.

The reduction is also made from 3-Dimensional Matching and also uses the same idea as Sitters [35] used to prove the strong NP-hardness of $\left\langle R|p m t n| \sum C_{j}\right\rangle$. In the reduction, the machines are thus also uniform machines with restricted availabilities. A complete proof can be found in the research report corresponding to this article [28].

\subsection{Lower Bound on the Competitiveness of Online Algorithms}

Muthukrishnan, Rajaraman, Shaheen, and Gehrke [33] propose an optimal online algorithm when there are only two job sizes. Mainly, they prove that there is no optimal online algorithm for the sum-stretch minimization problem when there are three or more distinct job sizes. Furthermore, they give a lower bound of 1.036 on the competitive ratio of any online algorithm. The following theorem improves this bound:

Theorem $6([27])$. No online algorithm minimizing the sum-stretch with preemption on a single processor has a competitive ratio less than or equal to 1.19484. 
Proof. Here we just present the adversary used to prove this lower bound. A complete proof can be found in the research report corresponding to this article [28]. The adversary behavior is defined by the following parameters: $\alpha=1.93716, \beta=1.29941, n=\frac{1}{\varepsilon} \approx 2.69598, k=10^{12}$, and $\varepsilon=0.370923$. Our adversary proceeds as follows:

1. At time $r_{0}=0$ we send a job $J_{0}$ of size $p_{0}=\alpha \beta n$.

2. At time $r_{1}=\alpha \beta n-\varepsilon$ we send a job $J_{1}$ of size $p_{1}=\beta n$.

3. We consider the system at time $\alpha \beta n+\beta n-\varepsilon$, and whether the execution of $J_{0}$ has been completed at that time.

(a) If the execution of $J_{0}$ has not yet been completed, we do not send any more jobs.

(b) Otherwise, at time $r_{2}=\alpha \beta n+\beta n-\varepsilon$ we send a job $J_{2}$ of size $p_{2}=n$.

We consider the system at time $\alpha \beta n+\beta n+n-\varepsilon$, and whether the execution of $J_{1}$ has been completed at that time.

i. If the execution of $J_{1}$ has not yet been completed, we do not send any more jobs.

ii. Otherwise, at time $r_{3}=\alpha \beta n+\beta n+n-\varepsilon$ we send a job $J_{3}$ of size $p_{3}=n$.

We consider the system at time $\alpha \beta n+\beta n+2 n-\varepsilon$, and whether the execution of $J_{2}$ has been completed at that time.

A. If the execution of $J_{2}$ has not yet been completed, we do not send any more jobs.

B. Otherwise, we send to the system a series of $k$ unit-size jobs, the inter-arrival time of these jobs being equal to their size: at time $r_{3+j}=\alpha \beta n+\beta n+2 n-\varepsilon+(j-1)$ we send the job $J_{3+j}$ of size $p_{3+j}=1$, for $1 \leqslant j \leqslant k$.

The parameters are chosen so that the optimal completion order of the jobs is $J_{1}, J_{2}, J_{3}, J_{3+1}, \ldots, J_{3+k}$, $J_{0}$.

\subsection{Shortest Processing Time Rules: SRPT, SWPT, SWRPT}

In the previous section, we have recalled that shortest remaining processing time (SRPT) is optimal for minimizing the sum-flow. When SRPT takes a scheduling decision, it only considers the remaining processing time of a job, and not its original processing time. Therefore, from the point of view of the sum-stretch minimization, SRPT does not take into account the weight of the jobs in the objective function. Nevertheless, Muthukrishnan, Rajaraman, Shaheen, and Gehrke have shown [33] that SRPT is 2-competitive for sum-stretch.

Another well studied algorithm is the Smith's ratio rule [37] also known as shortest weighted processing time (SWPT). This is a preemptive list scheduling where the available jobs are executed in increasing value of the ratio $\frac{p_{j}}{w_{j}}$. Whatever the weights, SWPT is 2-competitive [34] for the minimization of the sum of weighted completion times $\left(\sum w_{j} C_{j}\right)$. Note that a $\rho$-competitive algorithm for the sum weighted flow minimization $\left(\sum w_{j}\left(C_{j}-r_{j}\right)\right)$ is $\rho$-competitive for the sum weighted completion time $\left(\sum w_{j} C_{j}\right)$. However, the reverse is not true: a guarantee on the sum weighted completion time $\left(\sum w_{j} C_{j}\right)$ does not induce any guarantee on the sum weighted flow $\left(\sum w_{j}\left(C_{j}-r_{j}\right)\right)$. Therefore, the previous ratio on the minimization of the sum of weighted completion times gives us no result on the efficiency of SWPT for the minimization of the sum-stretch. Furthermore, we can even prove that SWPT is not an approximation algorithm for minimizing the sum-stretch. Indeed, SWPT schedules the available jobs by increasing values of $\frac{1}{p_{j}^{2}}$ and has thus exactly the same behavior as the shortest processing time first heuristic (SPT). The following theorem states that SPT (and thus SWPT) is not a competitive algorithm for minimizing the sum-stretch.

Theorem 7 ([27]). For any value $\rho>1$, there is an instance on which the sum-stretch realized by SPT is at least $\rho$ times the optimal. Furthermore, we can impose that in this instance $\Delta$, the ratio of the sizes of the largest and shortest jobs submitted to the system, is equal to 2.

Proof. Without loss of generality, we assume that $\rho$ is a strictly positive integer. Then, the problematic instance is made of $4 \rho+1$ jobs where job $J_{k}, 0 \leqslant k \leqslant 4 \rho$, is defined by: $r_{k}=8 \rho k-\frac{k(k+1)}{2}$ and $p_{k}=8 \rho-k$. A complete proof can be found in the research report corresponding to this article [28]. 
The weakness of the SWPT heuristics is obviously that it does not take into account the remaining processing times: it may preempt a job when it is almost completed. To address the weaknesses of both SRPT and SWPT, one might consider a heuristic that takes into account both the original and the remaining processing times of the jobs. This is what the shortest weighted remaining processing time heuristic (SWRPT) does. In the framework of sum-stretch minimization, at any time $t, \mathrm{SWRPT}$ schedules the job $J_{j}$ which minimizes $p_{j} \rho_{t}(j)$. Muthukrishnan, Rajaraman, Shaheen, and Gehrke [33] prove that SWRPT is actually optimal when there are only two job sizes.

Neither of the proofs of competitiveness of SRPT or SWPT can be extended to SWRPT. SWRPT has apparently been studied by Megow [31], but only in the scope of the sum weighted completion time. So far, there is no guarantee on the efficiency of SWRPT for sum-stretch minimization. Intuitively, we would think that SWRPT is more efficient than SRPT for the sum-stretch minimization. However, the following theorem shows that the worst case for SWRPT for the sum-stretch minimization is no better than that of SRPT.

Theorem 8 ([27]). For any real $\varepsilon, 1>\varepsilon>0$, there exists an instance such that SWRPT is not $(2-\varepsilon)$-competitive for the minimization of the sum-stretch.

Proof. Here we just present the construction used to prove this lower bound on the competitive ratio of SWRPT. A complete proof can be found in the research report corresponding to this article [28]. The problematic instance is composed of two sequences of jobs. In the first sequence, the jobs are of decreasing sizes, the size of a job being the square root of the size of its immediate predecessor. In the second sequence, all the jobs are of unit-size. Each job arrives at a date equal to the release date of its predecessor plus the execution time of this predecessor, except for the second and third jobs which arrive at dates critical for SWRPT.

Let $\alpha=1-\frac{\varepsilon}{3}, n=\left\lceil\log _{2}\left(\log _{2} \frac{3(1+\alpha)}{\varepsilon}\right)\right\rceil$, and $k=\left\lceil-\log _{2}\left(-\log _{2} \alpha\right)\right\rceil$. Let $l$ be an integer. Then, we formally build the instance $\mathcal{J}$ as follows:

1. Job $J_{0}$ arrives at time $r_{0}=0$ and is of size $p_{0}=2^{2^{n}}$.

2. Job $J_{1}$ arrives at time $r_{1}=2^{2^{n}}-2^{2^{n-2}}$ and is of size $p_{1}=2^{2^{n-1}}$.

3. Job $J_{2}$ arrives at time $r_{2}=r_{1}+2^{2^{n-1}}-\alpha$ and is of size $p_{2}=2^{2^{n-2}}$.

4. Job $J_{j}$, for $3 \leqslant j \leqslant n$, arrives at time $r_{j}=r_{j-1}+p_{j-1}$ and is of size $p_{j}=2^{2^{n-j}}$.

5. Job $J_{n+j}$, for $1 \leqslant j \leqslant k$, is of size $p_{n+j}=2^{2^{-j}}$ and arrives at time $r_{n+j}=r_{n+j-1}+p_{n+j-1}$.

6. Job $J_{n+k+j}$, for $1 \leqslant j \leqslant l$, is of size $p_{n+k+j}=1$ and arrives at time $r_{n+k+j}=r_{n+k+j-1}+p_{n+k+j-1}$.

Then, we evaluate the sum-stretch realized by both SWRPT and SRPT and we show that, if $l$ is large enough, the sum-stretch realized by SWRPT is $(\mathcal{R} \geqslant 2-\varepsilon)$-times that realized by SRPT. This proves the result as the optimal sum-stretch is no greater than that of SRPT.

\section{Offline Max-Stretch Optimization}

Bender, Chakrabarti, and Muthukrishnan [5] have shown that the problem of max-stretch minimization on one machine without preemption, i.e., problem $\left\langle 1\left|r_{j}\right| S_{\max }\right\rangle$, cannot be approximated within a factor $O\left(n^{1-\varepsilon}\right)$ for arbitrarily small $\varepsilon>0$, unless $\mathrm{P}=\mathrm{NP}$. In this section, we show that if we allow either divisible loads or preemptions, we are able to minimize the maximum weighted flow in polynomial time even on unrelated machines.

In Section 6.1, we state the relationship between minimization of the maximum weighted flow problem and deadline scheduling. Then we present a solution to maximum weighted flow minimization in the divisible load framework, on unrelated machines. By adapting some of these techniques, we then describe a solution to the minimization of the maximum weighted flow when preemption (but not load divisibility) is allowed, once again on unrelated machines. These results are given in Section 6.2.

It should be noted that, prior to our work, at least two solutions were known for minimizing the max-stretch on one machine with preemption. Baker, Lawler, Lenstra, and Rinnooy Kan [2] presented 
an $O\left(n^{2}\right)$ algorithm to solve an even more general problem: $\left\langle 1\left|p m t n, p r e c, r_{j}\right| f_{\max }\right\rangle$ (where $f_{\max }$ is the maximum of the costs of the jobs and the cost of a job is a non-decreasing function of its completion time). This algorithm determines the job of least priority and then iterates. Another solution using network flow maximization techniques was $\mathrm{known}^{2}$. In our divisible load framework, we do not know how to extend this flow maximization technique to solve the case of uniform machines with restricted availabilities, much less the more general case of unrelated processors. Nevertheless, for the sake of completeness, we recall this solution in Section 6.1.4. Finally, let us mention that the article [23] by Lawler and Labetoulle contains explicitly, or implicitly, most (if not all) of the techniques used in this section. We nevertheless fully expose the max-stretch minimization algorithm as these techniques may not be so widely known ${ }^{3}$ and as a good knowledge of the algorithm is necessary to understand the content of Sections 7 and 8 .

\subsection{Minimizing the Maximum Weighted Flow in the Divisible Model}

\subsubsection{Max Weighted Flow Minimization and Deadline Scheduling}

Let us assume that we are looking for a schedule $\mathcal{S}$ under which the maximum weighted flow is less than or equal to some objective value $\mathcal{F}$. The weighted flow of any job $J_{j}$ is equal to $w_{j}\left(C_{j}-r_{j}\right)$. Then, we should have:

$$
\max _{1 \leqslant j \leqslant n} w_{j}\left(C_{j}-r_{j}\right) \leqslant \mathcal{F} \quad \Leftrightarrow \quad \forall j \in[1 ; n], w_{j}\left(C_{j}-r_{j}\right) \leqslant \mathcal{F} \quad \Leftrightarrow \quad \forall j \in[1 ; n], C_{j} \leqslant r_{j}+\mathcal{F} / w_{j} .
$$

Thus, the execution of $J_{j}$ must be completed before time $d_{j}(\mathcal{F})=r_{j}+\mathcal{F} / w_{j}$ for schedule $\mathcal{S}$ to satisfy the bound $\mathcal{F}$ on the maximum weighted flow. Therefore, looking for a schedule which satisfies a given upper bound on the maximum weighted flow is equivalent to an instance of the deadline scheduling problem. We now show how to solve such a deadline scheduling problem in the divisible load framework.

In deadline scheduling, each job $J_{j}$ has not only a release date $r_{j}$ but also a deadline $d_{j}$. The problem is then to find a schedule such that each job $J_{j}$ is executed within its executable time interval $\left[r_{j}, d_{j}\right]$. We consider the set of all job release dates and deadlines: $\left\{r_{1}, \ldots, r_{n}, d_{1}, \ldots, d_{n}\right\}$. We define an epochal time as a time value at which one or more points in this set occur; there are between 2 (when all jobs are released at the same date and have the same deadline) and $2 n$ (when all job release dates and deadlines are distinct) such values. When ordered in absolute time, adjacent epochal times define a set of time intervals. We denote each time interval $I_{t}$ by $I_{t}=\left[\inf I_{t}, \sup I_{t}\left[\right.\right.$. Finally, we denote by $\alpha_{i, j}^{(t)}$ the fraction of job $J_{j}$ processed by machine $M_{i}$ during the time interval $I_{t}$. In this framework, System (1) lists the constraints that should hold true in any valid schedule:

a. release date: job $J_{j}$ cannot be processed before it is released (Equation (1a));

b. deadline: job $J_{j}$ cannot be processed after its deadline (Equation (1b));

c. resource usage: during a time interval, a machine cannot be used longer than the duration of this time interval (Equation (1c));

d. job completion: each job must be processed to completion (Equation (1d)).

$$
\left\{\begin{array}{l}
\text { (1a) } \forall i, \forall j, \forall t, \quad r_{j} \geqslant \sup I_{t} \Rightarrow \alpha_{i, j}^{(t)}=0 \\
\text { (1b) } \forall i, \forall j, \forall t, \quad d_{j} \leqslant \inf I_{t} \Rightarrow \alpha_{i, j}^{(t)}=0 \\
\text { (1c) } \forall t, \forall i, \quad \sum_{j} \alpha_{i, j}^{(t)} \cdot p_{i, j} \leqslant \sup I_{t}-\inf I_{t} \\
\text { (1d) } \quad \forall j, \quad \sum_{t} \sum_{i} \alpha_{i, j}^{(t)}=1
\end{array}\right.
$$

Lemma 2. System (1) has a solution if, and only if, there exists a solution to the deadline scheduling problem.

\footnotetext{
${ }^{2}$ We do not know any reference to this technique who was presented to us by Michael Bender.

${ }^{3}$ For instance, Bender, Chakrabarti, and Muthukrishnan proved in [5] the existence of a PTAS for a problem that is solved in polynomial-time in this section.
} 
System (1) can be solved in polynomial time by any linear solver system as all its variables are rational. Building a valid schedule from any solution of System (1) is straightforward as for any time interval $I_{t}$, and on any machine $M_{i}$, the job fractions $\alpha_{i, j}^{(t)}$ can be scheduled in any order.

One may think that by applying a binary search on possible values of the objective value $\mathcal{F}$, one would be able to find the optimal maximum weighted flow, and an optimal schedule. However, a binary search on rational values will not terminate. By setting a limit on the precision of the binary search, the number of process iterations is bounded, and the quality of the approximation can be guaranteed. However, as we now show, we can adapt our search to always find the optimal in polynomial time.

\subsubsection{Solving on a Range.}

So far we have used System (1) to check whether our problem has a solution whose maximum weighted flow is no greater than some objective value $\mathcal{F}$. We now show that we can use it to check whether our problem has a solution for some particular range of objective values. Later we show how to divide the whole search space into a polynomial number of search ranges.

First, let us suppose there exist two values $\mathcal{F}_{1}$ and $\mathcal{F}_{2}, \mathcal{F}_{1}<\mathcal{F}_{2}$, such that the relative order of the release dates and deadlines, $r_{1}, \ldots, r_{n}, d_{1}(\mathcal{F}), \ldots, d_{n}(\mathcal{F})$, when ordered in absolute time, is independent of the value of $\mathcal{F} \in] \mathcal{F}_{1} ; \mathcal{F}_{2}[$. Then, on the objective interval $] \mathcal{F}_{1}, \mathcal{F}_{2}[$, as before, we define an epochal time as a time value at which one or more points in the set $\left\{r_{1}, \ldots, r_{n}, d_{1}(\mathcal{F}), \ldots, d_{n}(\mathcal{F})\right\}$ occurs. Note that an epochal time which corresponds to a deadline is no longer a constant but an affine function in $\mathcal{F}$. As previously, when ordered in absolute time, adjacent epochal times define a set of time intervals, that we denote by $I_{1}, \ldots, I_{n_{\text {int }}(\mathcal{F})}$. The durations of time intervals are now affine functions in $\mathcal{F}$. Using these new definitions and notations, we can solve our problem on the objective interval $\left[\mathcal{F}_{1}, \mathcal{F}_{2}\right]$ using System (1) with the additional constraint that $\mathcal{F}$ belongs to $\left[\mathcal{F}_{1}, \mathcal{F}_{2}\right]\left(\mathcal{F}_{1} \leqslant \mathcal{F} \leqslant \mathcal{F}_{2}\right)$, and with the minimization of $\mathcal{F}$ as the objective. This gives us System (2).

$$
\begin{aligned}
& \text { Minimize } \mathcal{F}, \\
& \text { Under the COnstraints } \\
& \begin{cases}(2 \mathrm{a}) \quad \forall i, \forall j, \forall t, \quad r_{j} \geqslant \sup I_{t} \Rightarrow \alpha_{i, j}^{(t)}=0 \\
(2 \mathrm{~b}) \quad \forall i, \forall j, \forall t, \quad d_{j} \leqslant \inf I_{t} \Rightarrow \alpha_{i, j}^{(t)}=0 \\
(2 \mathrm{c}) \quad \forall t, \forall i, \quad \sum_{j} \alpha_{i, j}^{(t)} \cdot p_{i, j} \leqslant \sup I_{t}-\inf I_{t} \\
(2 \mathrm{~d}) \quad \forall j, \quad \sum_{t} \sum_{i} \alpha_{i, j}^{(t)}=1 \\
(2 \mathrm{e}) \quad \mathcal{F}_{1} \leqslant \mathcal{F} \leqslant \mathcal{F}_{2}\end{cases}
\end{aligned}
$$

\subsubsection{Particular Objectives.}

The relative ordering of the release dates and deadlines only changes for values of $\mathcal{F}$ where one deadline coincides with a release date or with another deadline. We call such a value of $\mathcal{F}$ a milestone ${ }^{4}$. In our problem, there are at most $n$ distinct release dates and as many distinct deadlines. Thus, there are at most $\frac{n(n-1)}{2}$ milestones at which a deadline function coincides with a release date. There are also at most $\frac{n(n-1)}{2}$ milestones at which two deadline functions coincides (two affine functions intersect in at most one point). Let $n_{\mathrm{q}}$ be the number of distinct milestones. Then, $1 \leqslant n_{\mathrm{q}} \leqslant n^{2}-n$. We denote by $\mathcal{F}_{1}, \mathcal{F}_{2}, \ldots, \mathcal{F}_{n_{\mathrm{q}}}$ the milestones ordered by increasing values. To solve our problem we just need to perform a binary search on the set of milestones $\mathcal{F}_{1}, \mathcal{F}_{2}, \ldots, \mathcal{F}_{n_{\mathrm{q}}}$, each time checking whether System (2) has a solution in the objective interval $\left[\mathcal{F}_{i}, \mathcal{F}_{i+1}\right]$ (except for $i=n_{\mathrm{q}}$ in which case we search for a solution in the range $\left[\mathcal{F}_{n_{\mathrm{q}}},+\infty[\right.$ ). There is a polynomial number of milestones and System (2) can be solved in polynomial time. Therefore:

Theorem 9 ([26]). The problem of minimizing the maximum weighted flow time in the divisible load model $\langle R| r_{j} ;$ div $\left|\max w_{j} F_{j}\right\rangle$ can be solved in polynomial time.

\footnotetext{
${ }^{4}$ Labetoulle, Lawler, Lenstra, and Rinnooy Kan [22] call such a value a "critical trial value".
} 


\subsubsection{A Network Flow Approach for Uniform Machines}

In section 6.1.1, we presented Linear program 1 to check whether there exists a schedule whose maximum weighted flow is no greater than a given objective. This linear program solves this problem in the unrelated machines case, that is, the most general one. In fact, in the uniform machines framework, one can solve this problem using a network flow maximization approach. The graph is built as follows:

Vertices. The graph contains:

- A source;

- A sink;

- One vertex $J_{j}$, for each job $J_{j}, 1 \leqslant j \leqslant n$;

- One vertex $\left(I_{t}, M_{i}\right)$ for each ordered pair made of a time interval $I_{t}, 1 \leqslant t \leqslant n_{\text {int }}$, and of a machine $M_{i}, 1 \leqslant i \leqslant m$.

Edges. The graph contains:

- One edge from the source to each node $J_{j}$ of capacity $W_{j}$, the size of the job. This edge represents the amount of work that must be done for the job $J_{j}$.

- One edge from each node $J_{j}$ to each node $\left(I_{t}, M_{i}\right)$ if, and only if, job $J_{j}$ can be executed during the time interval $I_{t}$ (i.e., $r_{j} \leqslant \inf I_{t}$ and $\sup t \leqslant d_{j}$ ). This edge is also of capacity $W_{j}$ (and is thus not constraining).

- One edge from each node $\left(I_{t}, M_{i}\right)$ to the sink, of capacity $\frac{\sup I_{t}-\inf I_{t}}{c_{i}}$ : this is the amount of work that machine $M_{i}$ can perform during the time interval $I_{t}$.

Figure 3 presents an example of such a graph.

There exists a schedule whose maximum weighted flow is no greater than a given objective $\mathcal{F}$ if the network flow maximization problem for the graph defined above (for the time intervals corresponding to $\mathcal{F}$ ) has a solution whose flow is equal to $\sum_{j} W_{j}$. As previously, one can just check the feasibility of the network flow problem for the milestones defined in the previous section. Then, when it is known between which two milestones lies the optimal, the ordering of deadlines is known, and an Earliest Deadline First scheduling leads to an optimal solution. However, this scheme only works in the uniform machines setting as EDF is no longer optimal for uniform machines with restricted availabilities (see the example of Figure 2). Therefore, we do not know how to use the network flow approach to minimize the max-stretch on uniform machines with restricted availabilities, but this approach can obviously be used in such a framework to check whether a given objective is feasible. Furthermore, this network flow approach cannot be straightforwardly extended to deal with the general case of unrelated machines (even for the problem of uniform machines with restricted availabilities).

\subsection{Minimizing the Maximum Weighted Flow with Preemption (but no Di- visibility)}

In this section, we focus on the more classical problem with preemption but without the divisible load assumption. We show that combining the linear programming approach of the previous section with the work of Lawler and Labetoulle [23] leads to a polynomial-time algorithm to solve this problem on unrelated machines. Note that the network flow approach we just recalled enables to minimize the max-stretch with preemption on one machine.

Following the work of Gonzalez and Sahni [19], Lawler and Labetoulle [23] present a scheme to build in polynomial-time a preemptive schedule of makespan $\mathcal{C}$ for a set of jobs $J_{1}, \ldots, J_{n}$ of null release dates $\left(\forall j, r_{j}=0\right)$, under the condition that Linear System (3) has a solution. This system simply states that:

a. all jobs must be fully processed (Equation (3a));

b. the whole processing of a job cannot take a time larger than $\mathcal{C}$ (Equation (3b));

c. the whole utilization time of a machine cannot be longer than a time $\mathcal{C}$ (Equation (3c)). 


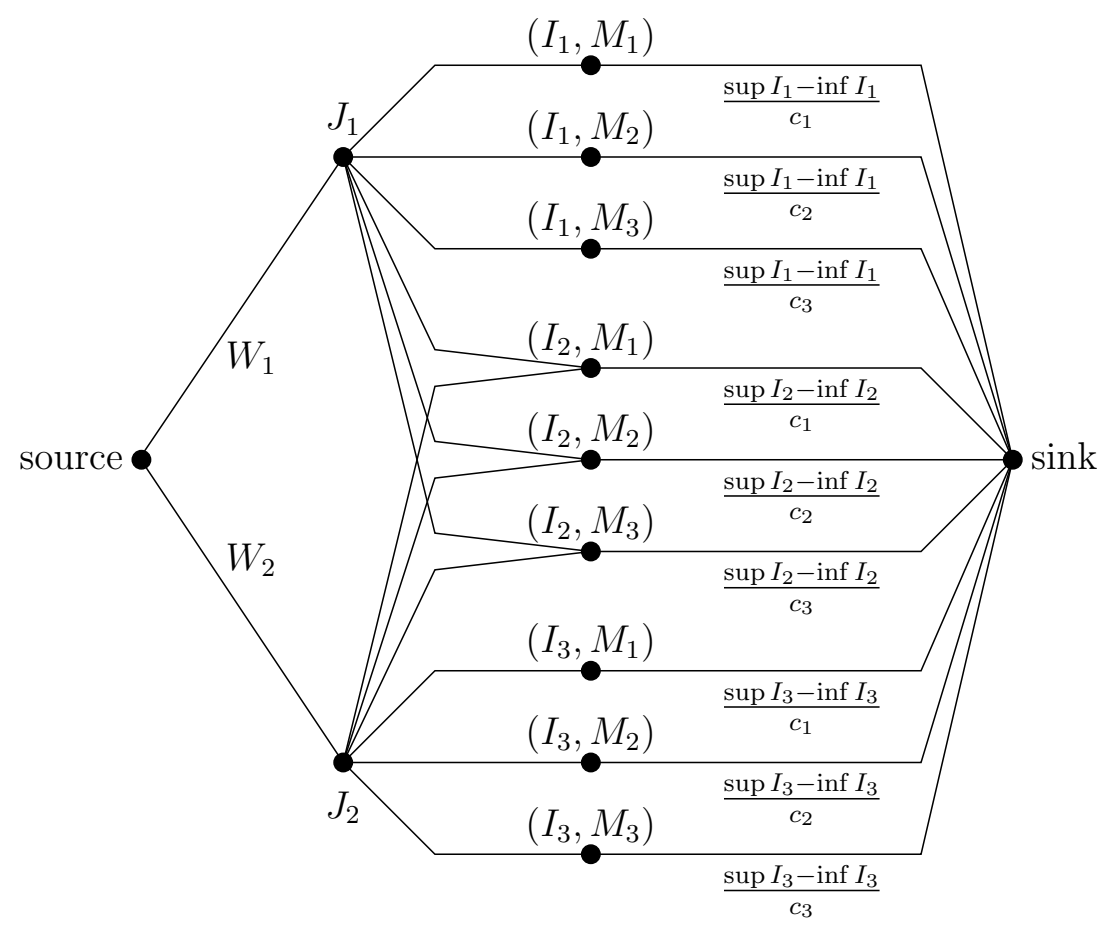

Figure 3: Graph used to check, on uniform machines and through network flow maximization, whether there exists a schedule of a given maximum weighted flow. This example has two jobs, three machines, and three time intervals defined by the epochal times $r_{1}<r_{2}<d_{1}<d_{2}$.

Obviously, these constraints must be satisfied by any preemptive schedule whose makespan is no longer than $\mathcal{C}$. The constructive result obtained by Lawler and Labetoulle shows that such a schedule exists if, and only if, this set of constraints has a solution.

$$
\begin{cases}(3 \mathrm{a}) \quad \forall j, \quad \sum_{i=1}^{m} \alpha_{i, j}=1 \\ (3 \mathrm{~b}) \quad \forall j, \quad \sum_{i=1}^{m} \alpha_{i, j} \cdot p_{i, j} \leqslant \mathcal{C} \\ (3 \mathrm{c}) \quad \forall i, \quad \sum_{j=1}^{n} \alpha_{i, j} \cdot p_{i, j} \leqslant \mathcal{C}\end{cases}
$$

Our problem is slightly more general in that we allow arbitrary release dates. Additionally, our objective is to minimize the maximum weighted flow rather than the makespan. Let us consider a maximum weighted flow objective $\mathcal{F}$. As we did in Section 6.1.1, we use this objective value to define for each job $J_{j}$ a deadline $d_{j}(\mathcal{F})=r_{j}+\mathcal{F} / w_{j}$. As before, the set of release dates and deadlines defines a set of epochal times which, in turn, defines a set of time intervals that we denote by $I_{1}, \ldots, I_{n_{\text {int }}(\mathcal{F})}$. Then, we claim that there exists a preemptive schedule whose maximum weighted flow is no greater than $\mathcal{F}$ if, and only if, Linear System (4) has a solution. Linear System (4) simply states that:

a. each job must be processed to completion (Equation (4a) which corresponds to Equation (3a));

b. the processing of a job during the time interval $I_{t}$ cannot take a time larger than the length of $I_{t}$ as, in the current framework, a job cannot be simultaneously processed by two different machines (Equation (4b) which corresponds to Equation (3b));

c. the utilization of a machine during a time interval cannot exceed its capacity (Equation (4c) which corresponds to Equation (3c)); 
d. the processing of a job cannot start before it is released (Equation (4d));

e. a job must be processed before its deadline (Equation (4e)).

$$
\begin{cases}(4 \mathrm{a}) \quad \forall j, \quad \sum_{t} \sum_{i} \alpha_{i, j}^{(t)}=1 \\ (4 \mathrm{~b}) \quad \forall t, \forall j, \quad \sum_{i} \alpha_{i, j}^{(t)} \cdot p_{i, j} \leqslant \sup I_{t}-\inf I_{t} \\ (4 \mathrm{c}) \quad \forall t, \forall i, \quad \sum_{j} \alpha_{i, j}^{(t)} \cdot p_{i, j} \leqslant \sup I_{t}-\inf I_{t} \\ (4 \mathrm{~d}) \quad \forall i, \forall j, \forall t, \quad r_{j} \geqslant \sup I_{t} \Rightarrow \alpha_{i, j}^{(t)}=0 \\ (4 \mathrm{e}) \quad \forall i, \forall j, \forall t, \quad d_{j} \leqslant \inf I_{t} \Rightarrow \alpha_{i, j}^{(t)}=0\end{cases}
$$

Any preemptive schedule whose maximum weighted flow is no greater than $\mathcal{F}$ must obviously satisfy Linear System (4). Conversely, suppose that Linear System (4) has a solution. Then, following Lawler and Labetoulle [23], we note that the whole system effectively decomposes into a set of linear subsystems, one for each of the time intervals, and that the sub-system corresponding to interval $I_{t}$ is exactly equivalent to Linear System (3) where the objective is the length of the time interval (i.e., $\mathcal{C}=$ $\sup I_{t}-\inf I_{t}$ ). Therefore, starting from a solution of Linear System (4) we use the polynomial-time reconstruction scheme of Lawler and Labetoulle to build a preemptive schedule for each of the time intervals $I_{t}$. The concatenation of these partial schedules gives us a solution to our problem.

Thus far, we have shown that we are able to check the feasibility of a specific objective value for maximum weighted flow in polynomial time. Moreover, if such an objective is feasible a schedule that achieves this maximum weighted flow can also be built in polynomial time. To finally solve our problem, we recall the methodology presented in Section 6.1: Linear System (4) can be used to search for a solution in a range of objective values, defined by consecutive milestones, over which the linear system is valid (i.e., the relative order of release dates and deadlines does not change). Similarly, a binary search over the milestones - which are in polynomial number - enables us to find and build an optimal solution in polynomial time. Therefore:

Theorem 10. The problem of minimizing the maximum weighted flow time in the single processor with preemption model $\langle R| r_{j} ;$ pmtn $\left|\max w_{j} F_{j}\right\rangle$ can be solved in polynomial time.

\section{Pareto Minimization of Max-Stretch and Max Flow}

In this section we present a few game theory notions and how they translate to our context. This enables us to understand a major flaw of the previous max-based metric in a general framework and how to correctly define a new metric.

Game theory provides a general framework to model situations where many users compete for resources. Each user (in our context, a job) is characterized by a utility function $u_{j}$. The utility functions represent the satisfaction perceived by the user (typically function of the delay or of the capacity). The goal is to find scheduling strategies such that the utility of each user is maximized. In our context it is more relevant to consider cost functions rather than utility functions. Indeed, scheduling problems are typically minimization problems as we try to minimize the completion time, the flow or the stretch of each job. We will therefore assume in the following that the cost $\gamma_{j}$ of job $J_{j}$ is a function of the completion times $C$. However, as these users may compete for the same resources, it is generally not possible to simultaneously minimize the cost of each user. In a multi-user context, optimality is not defined as simply as in the single-user context, and it is common to use Pareto-optimality, defined as follows:

Definition 2 (Pareto-optimality). A vector of completion times $C=\left(C_{1}, \ldots, C_{n}\right)$ is Pareto-optimal if and only if:

$$
\forall \widetilde{C}, \exists i, \gamma_{i}(\widetilde{C})<\gamma_{i}(C) \Rightarrow \exists j, \gamma_{j}(C)<\gamma_{j}(\widetilde{C})
$$


In other words, $C$ is Pareto optimal if it is impossible to strictly decrease the cost of a player without strictly increasing that of another. Any non-Pareto-optimal schedule can thus be considered as nonefficient as strictly a better usage of resources could be done. Let us consider the cost set $\Gamma \subseteq\left(\mathbb{R}_{+}^{*}\right)^{n}$ defined as the set of all feasible cost vectors:

$$
\Gamma=\left\{\left(\gamma_{1}(C), \ldots, \gamma_{n}(C) \mid \text { there exist a valid schedule with completion times } C\right\}\right.
$$

Figure 4 depicts on each subfigure, for a simple scheduling instance the various cost sets associated to the completion time, flow time and stretch metrics. The dashed-dotted line is the optimal isoline for

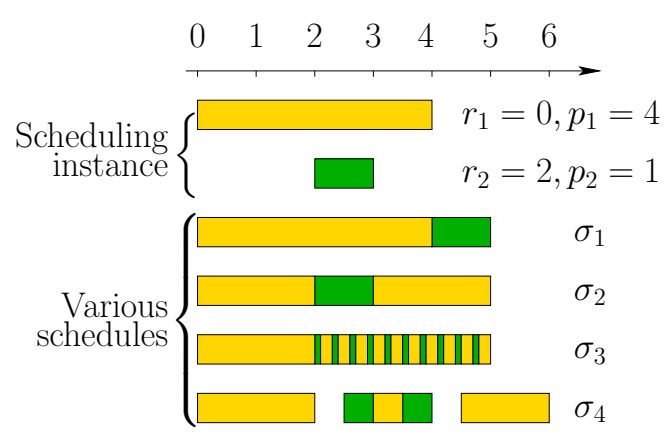

(a) Scheduling instance and various feasible schedules.

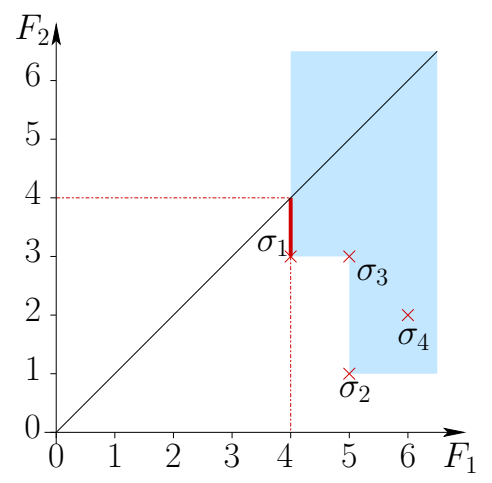

(c) Cost set for $F_{j} . \sigma_{1}$ and any schedule such that $F_{1}$ and $F_{2}$ are smaller than 4 is optimal for $\max _{j} F_{j}$. However, only $\sigma_{1}$ is Pareto-optimal for $\max _{j} F_{j}$.

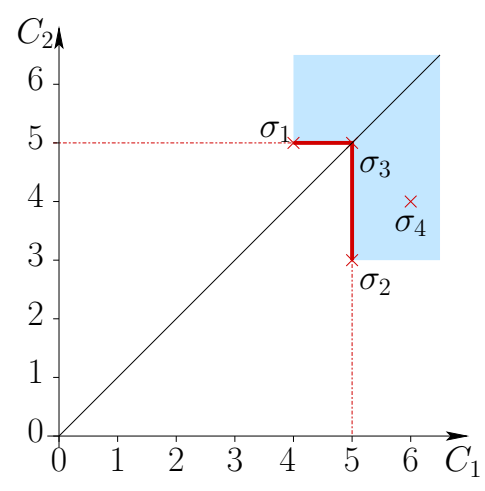

(b) Cost set for $C_{j}$. Bold lines depict optimal schedule (hence $\sigma_{1}, \sigma_{2}$ and $\sigma_{3}$ ) for the $\max _{j} C_{j}$ metric. However, only $\sigma_{1}$ and $\sigma_{2}$ are Pareto-optimal.

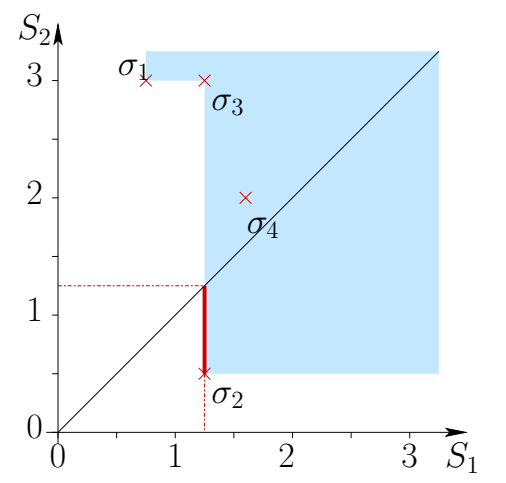

(d) Cost set for $S_{j} . \sigma_{2}$ and any schedule such that $S_{1}$ and $S_{2}$ are smaller than 1.25 is optimal for $\max _{j} S_{j}$. However, only $\sigma_{2}$ is Pareto-optimal for $\max _{j} S_{j}$.

Figure 4: Most optimal solutions to max-based metrics are not Pareto-optimal.

the considered max-based metric $\left(\max _{j} C_{j}\right.$ for Figure $4(\mathrm{~b}), \max _{j} F_{j}$ for Figure $4(\mathrm{c})$, and $\max _{j} S_{j}$ for Figure 4(d)). Any point (the bold lines) belonging to both the isoline and the cost set is thus optimal for the max-based metric. However we can see that very few of them are Pareto-optimal. This is due to the fact that only the first maximum has been minimized. It is well-known in the network community (see for example $[8,30])$ that max-min fairness should be recursively defined. In our setting, this means that the first maximum should be minimized, then the second should be minimized, and so on. Sum-based metrics obviously do not suffer from this flaw and always produce Pareto-optimal schedules. That is why we propose to consider the new metrics $C_{\max }$ Pareto, $F_{\max }$ Pareto, and $S_{\max }$ Pareto. These scheduling metrics are likely to be much more difficult (but also much more meaningful) than the previous ones as we do not have to only optimize the cost of the more constraining job but to optimize the cost of all jobs at the same time. 


\subsection{Heuristic Pareto Minimization of Max-Stretch on One Machine}

Algorithm 2 is an obvious algorithm which recursively tries to minimize the stretch of jobs: first it minimizes the max-stretch, then the number of jobs whose stretch is equal to the max-stretch, then the maximum stretch of the other jobs, and so on. This algorithm relies on the fact that Earliest Deadline First (EDF) always finds a schedule satisfying all deadlines if one exists $[16]^{5}$. We show that in some cases Algorithm 2 produces Pareto optimal schedules for stretch minimization.

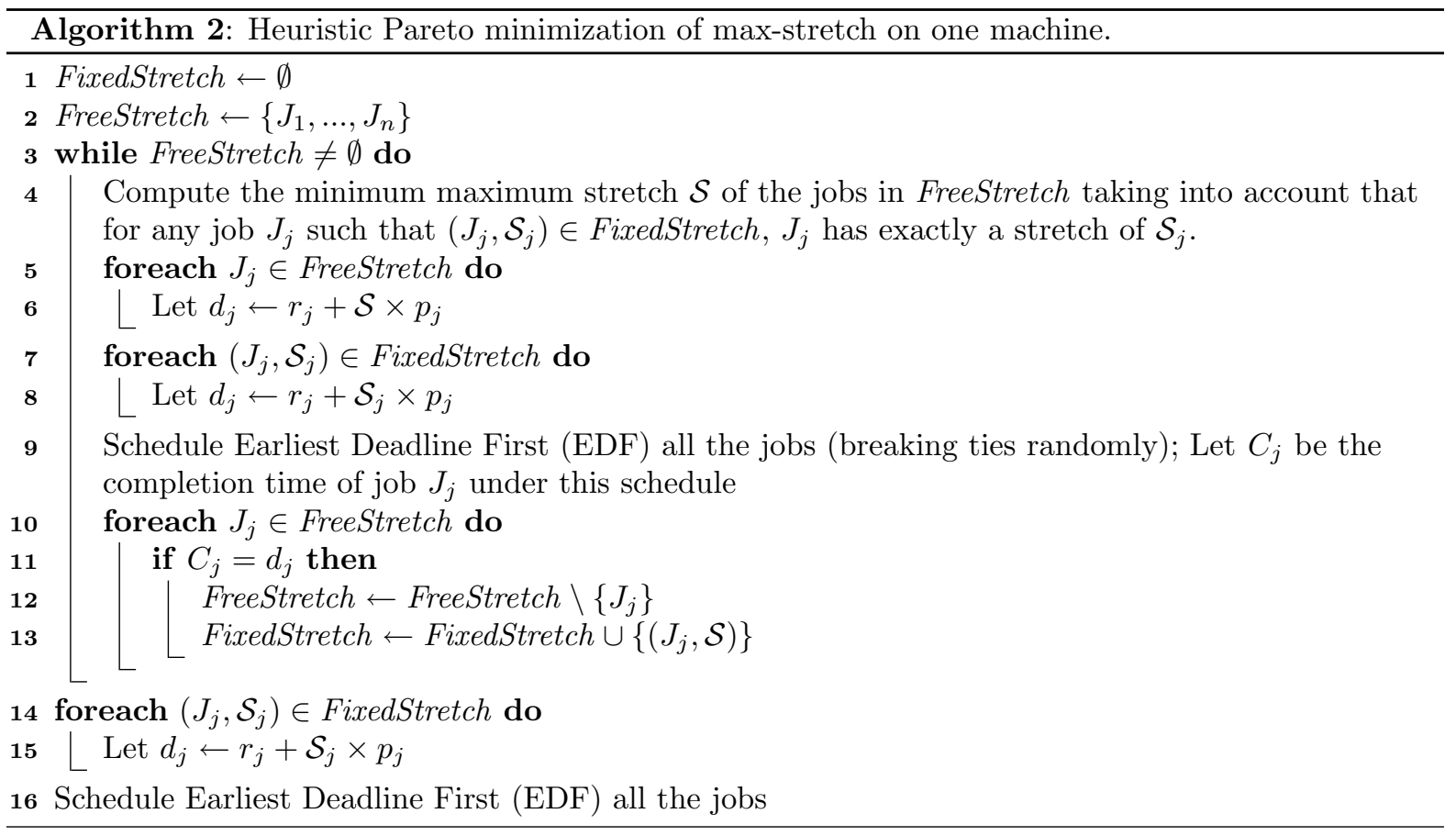

Theorem 11. Algorithm 2 presented below produces a Pareto optimal schedule for max-stretch minimization on one machine with preemption if at no iteration of the while loop there are two jobs whose deadlines, defined at Steps 6 or 8, are equal.

Proofs and details can be found in the research report corresponding to this article [28]. We conjecture that Algorithm 2 always produces a Pareto optimal schedule for max-stretch minimization on one machine with preemption. This conjecture is based on the facts that 1) the function which associates to a schedule the vector of the stretch of the jobs, sorted in non-decreasing order, is a continuous function; 2) we believe that the set of the instances for which Theorem 11 holds is dense in the space of all instances.

\subsection{Heuristic Pareto Minimization of Max Weighted Flow on Unrelated Ma- chines}

Here, we target the more general case of the max weighted flow as we will need to look at the special case of max-flow minimization.

Algorithm 3 presents the solution we propose for the general case. The solution for single processor case cannot be straightforwardly extended to the general case as the Earliest Deadline First algorithm is obviously not optimal for non-uniform machines. Once again we (try to) recursively optimize the max weighted flow of the jobs. We compute the best achievable max weighted flow for the jobs whose weighted flow is not yet fixed, and we (try to) minimize the number of jobs whose weighted flow is equal to this maximum. As always the objective max weighted flow gives a deadline per FreeWeightedFlow job, i.e., per job whose weighted flow has not yet been fixed. We first minimize the number of distinct deadlines $d$ such that there always is a job whose deadline is $d$ and which is completed at date $d$. Then we minimize the number of (problematic) jobs, i.e., of jobs which are completed at their deadline.

\footnotetext{
${ }^{5}$ On one processor, this property can even be extended to the case of tasks with general dependence relations [12].
} 


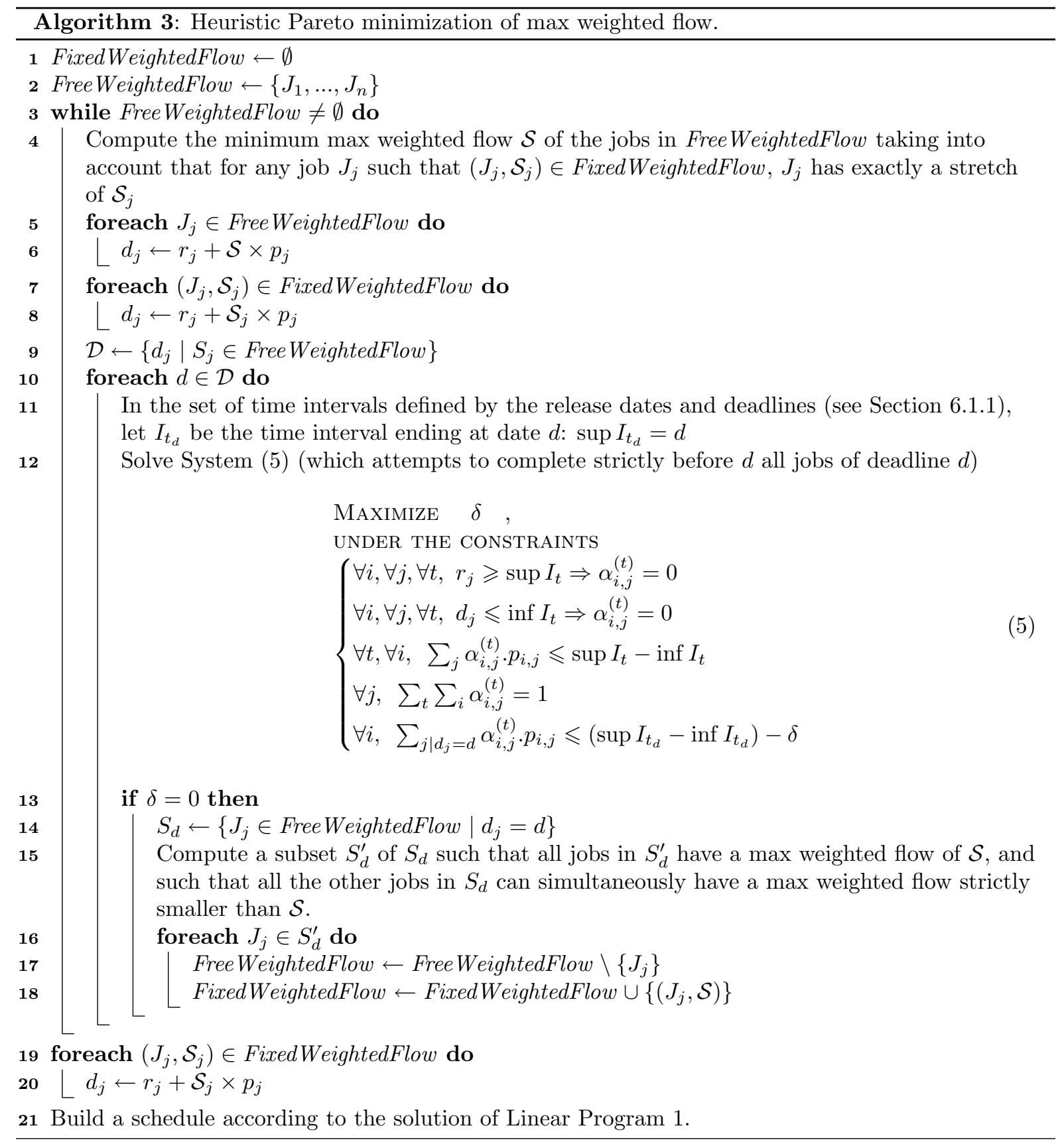


We can show that Algorithm 3 is correct.

Lemma 3. Algorithm 3 produces a valid schedule.

Proofs and details can be found in the research report corresponding to this article [28].

Step 15 does not explicit how the set " $S_{d}^{\prime}$ " of jobs whose completion date equals the deadline should be computed, especially as we would like this set to be as small as possible. In fact, in the general case this problem is NP-complete. The next theorem states the complexity of the Pareto max-flow minimization, and thus of the general case.

Theorem 12. The Pareto minimization of max-flow on unrelated machines, $\langle R|$ div $\mid F_{\max }$ Pareto $\rangle$, is NP-complete.

As we do not have any release dates in the above theorem, we in fact prove that $\left\langle R|\operatorname{div}| C_{\max }\right.$ Pareto $\rangle$, is NP-complete. In fact we prove an even stronger result, that is that minimizing the number of jobs whose completion date is equal to the makespan is NP-complete on unrelated machines, and under the divisible model. This result is proved with a reduction from Minimum Hitting Set [18]. The proof can be found in the research report corresponding to this article [28]

Minimum hitting Set is equivalent to Minimum Set cover [17]. Therefore, one of the best polynomial time algorithm to approximate Minimum HITTING SET is the greedy algorithm which at each step picks the element which belongs to the largest number of still un-hit subsets. This greedy algorithm has an approximation ratio of $1+\ln |S|[21,36]$, where $|S|$ is the size of the set.

We do not know what is the complexity of the Pareto minimization of the max-stretch. Seeing how efficient is the greedy heuristic for the minimum hitting set problem, we simply suggest to use it to solve in practice Step 15. Furthermore, one can easily see that when the set $S_{d}$ at Step 14 is always reduced to a singleton, Algorithm 3 produces an optimal schedule. Therefore:

Theorem 13. Algorithm 3 produces a Pareto optimal schedule for max weighted flow minimization on unrelated machines under the divisible load model if the set $S_{d}$ at Step 14 is always reduced to a singleton.

We believe that, in practice, the set $S_{d}$ will always be reduced to a singleton, and thus that Algorithm 3 will always produce optimal schedules in practice. (Note that the case of jobs of same size and same release date is not a problem.)

\section{Online Max-Stretch Optimization}

In this section, we first improve a lower bound on the competitive ratio of online algorithms for maxstretch minimization established by Bender, Chakrabarti, and Muthukrishnan [5]. Then we present the two competitive algorithms that have previously been proposed in the literature $[5,6]$. Last we highlight some practical limitations of these algorithms and propose new heuristics that circumvent these limitations.

\subsection{Lower Bound on the Competitiveness of Online Algorithms}

Theorem 14 ([27]). There is no $\frac{1}{2} \Delta^{\sqrt{2}-1}$-competitive preemptive online algorithm minimizing maxstretch if we restrict to instance with at least three different processing times.

This result is an improvement from the bound of $\frac{1}{2} \Delta^{\frac{1}{3}}$ established by Bender, Chakrabarti, and Muthukrishnan [5]. In fact, we establish this new bound by doing a more precise analysis of the exact same adversary. In their proof, Bender, Chakrabarti, and Muthukrishnan implicitly assumed that the algorithm knew in advance the ratio $\Delta$ of the sizes of the largest and shortest jobs. We will see in the next section that there exist some $O(\sqrt{\Delta})$-competitive algorithms. Therefore, we have roughly bridged half of the gap between the previous lower bound and the best existing algorithms.

Proof. We prove this result by contradiction. Therefore, let us assume that there exists a $\frac{1}{2} \Delta^{\sqrt{2}-1}$ competitive preemptive online algorithm $\mathcal{A}$ minimizing max-stretch. An adversary sends the following series of jobs:

Phase 1: Jobs 1 and 2 have both a size of $\delta$ and arrive at time 0 , i.e., $p_{1}=p_{2}=\delta$ and $r_{1}=r_{2}=0$. 
Phase 2: Starting at time $2 \delta-k$, and every $k$ time units, a job of size $k$ (with $k<\delta$ ) arrives. There are $x$ such jobs. In other words, for $1 \leqslant j \leqslant x$, job $J_{2+j}$ arrives at time $r_{2+j}=2 \delta+(j-2) k$ and is of size $p_{2+j}=k$.

A first come, first served (FCFS) ordering of all the jobs has a stretch of 2 . Algorithm $\mathcal{A}$ is by hypothesis $\frac{1}{2} \Delta^{\sqrt{2}-1}$-competitive and, as a stretch of 2 can be achieved, the date $C_{1}$ at which the execution of $J_{1}$ ends must satisfy: $\frac{C_{1}-r_{1}}{p_{1}} \leqslant \frac{1}{2} \Delta^{\sqrt{2}-1} \times 2$ (same constraint on $C_{2}$ ). So far, $\Delta=\frac{\delta}{k}$ (remember that $\Delta$ is the ratio of the sizes of the largest and shortest jobs in the system) ${ }^{6}$. Therefore, the constraint on $C_{1}$ can be rewritten:

$$
C_{1} \leqslant \frac{1}{2} \Delta^{\sqrt{2}-1} \times 2 \times \delta=\frac{\delta^{\sqrt{2}}}{k^{\sqrt{2}-1}} .
$$

The most favorable case for algorithm $\mathcal{A}$ is when it is able to (partially) delay the execution of $J_{1}$ and $J_{2}$ and to execute each of the jobs $J_{3}, \ldots, J_{2+x}$ at its release date. To forbid such a behavior, we choose $x$, the number of jobs of size $k$, to be large enough for $\mathcal{A}$ not to be able to delay the completion of $J_{1}$ and/or $J_{2}$ after the completion of all the jobs of size $k$. If each of the jobs $J_{3}, \ldots, J_{2+x}$ is executed at its release date, then $C_{2+x}=2 \delta+(x-1) k$. We define $x$ as follows:

$$
x=\left\lfloor 2+\left(\frac{\delta}{k}\right)^{\sqrt{2}}-\frac{2 \delta}{k}\right\rfloor .
$$

Then the execution of $C_{1}$ and $C_{2}$ must be completed by the date $2 \delta+(x-1) k$. Otherwise, the algorithm $\mathcal{A}$ fails to achieve its guarantee as the adversary would then send at time $2 \delta+(x-1) k$ a job of size $\frac{k+\delta}{2}$ to be exactly under the conditions stated by the theorem. So, algorithm $\mathcal{A}$ must complete the execution of $C_{1}$ and $C_{2}$ by the date $2 \delta+(x-1) k$. Then the adversary sends the following third series of jobs.

Phase 3: Starting at time $2 \delta+(x-1) k$, and every time unit, arrives a job of size 1 . There are $y$ such jobs. In other words, for $1 \leqslant j \leqslant y$, job $J_{2+x+j}$ arrives at time $r_{2+x+j}=2 \delta+(x-1) k+(j-1)$ and is of size $p_{2+x+j}=1$.

The optimal max-stretch is then less than or equal to $\frac{2 \delta+x k+y}{\delta}$ (obtained when delaying the completion of $J_{1}$ or $J_{2}$ after the completion of all smaller jobs). The max-stretch that algorithm $\mathcal{A}$ can achieve is greater than or equal to $k+1$ when we let $y=\lceil k(k-1)\rceil$. Indeed, the last job completed by algorithm $\mathcal{A}$ is either of size 1 or $k$ and, whatever its size, its stretch is thus the max-stretch of $\mathcal{A}$. Finally, when we pick $k=\delta^{2-\sqrt{2}}$, we obtain the desired contradiction on the competitive ratio of Algorithm $\mathcal{A}$.

\subsection{Competitive Online Heuristics}

We have already seen in Section 3.2 that FCFS, the optimal algorithm for the online minimization of max-flow, is only $\Delta$-competitive for the online minimization of max-stretch. This seemingly bad result is obviously partially explained by Theorem 14 .

We now recall two existing online algorithms for max-stretch minimization before introducing a new one. Bender, Muthukrishnan, and Rajaraman [6] defined, for any job $J_{j}$, a pseudo-stretch $\widehat{\mathcal{S}}_{j}(t)$ :

$$
\widehat{\mathcal{S}}_{j}(t)= \begin{cases}\frac{t-r_{j}}{\sqrt{\Delta}} & \text { if } 1 \leqslant p_{j} \leqslant \sqrt{\Delta}, \\ \frac{t-r_{j}}{\Delta} & \text { if } \sqrt{\Delta}<p_{j} \leqslant \Delta .\end{cases}
$$

Then, they scheduled the jobs by decreasing pseudo-stretches, potentially preempting running jobs each time a new job arrives in the system. They demonstrated that this method is a $O(\sqrt{\Delta})$-competitive online algorithm.

Bender, Chakrabarti, and Muthukrishnan [5] had previously described another $O(\sqrt{\Delta})$-competitive online algorithm for max-stretch. This algorithm works as follows: each time a new job arrives, the currently running job is preempted. Then, they compute the optimal (offline) max-stretch $\mathcal{S}^{*}$ of all

\footnotetext{
${ }^{6}$ Our bound is tighter than the one established by Bender, Chakrabarti, and Muthukrishnan because we remark that $\Delta=\frac{\delta}{k}$, when they used $\Delta=\frac{\delta}{1}=\delta$, as if they had assumed that the algorithm knew in advance that the ratio of the sizes of the largest and shortest jobs submitted to the system would be $\delta$.
} 
jobs having arrived up to the current time. Next, a deadline is computed for each job $J_{j}: d_{j}(\mathcal{F})=$ $r_{j}+\alpha \times \mathcal{S}^{*} / p_{j}$ Finally, a schedule is realized by executing jobs according to their deadlines, using the Earliest Deadline First strategy. To optimize their competitive ratio, Bender et al. set their expansion factor $\alpha$ to $\sqrt{\Delta}$. For both heuristics, the ratio $\Delta$ of the sizes of the largest and shortest jobs submitted to the system is thus assumed to be known in advance.

When they designed their algorithm, Bender et al. did not know how to compute the (offline) optimal maximum stretch. This problem is now overcome. The main remaining problem in this approach, from our point of view, is that such an algorithm tries only to optimize the stretch of the most constraining jobs. This problem is common to all algorithms minimizing a max objective. Indeed, such an algorithm may very easily schedule all jobs so that their stretch is equal to the objective, even if most of them could have been scheduled to achieve far lower stretches. This problem is far from being merely theoretical, as we will see in Section 9. We will try to circumvent it when designing our own heuristics.

\subsection{Practical Online Heuristics}

The basic online heuristic we could derive from our offline algorithm would be along the same line as the algorithm of Bender, Chakrabarti, and Muthukrishnan: each time a new job arrives we would preempt the running job (if any), compute the optimal max-stretch, and schedule the jobs according to the solution of System 2. The solution of System 2 specifies what fraction of each job should be executed on each processor during each time interval. We would implement this solution by breaking arbitrarily the ties that may appear in each time interval.

Our first modification to this scheme is that, rather than computing the "optimal max-stretch", we compute the "best achievable max-stretch considering the decisions already made". In other words, we take into account our knowledge of which jobs were already (partially) executed, and when. The underlying idea being that we cannot change the past. Also, such an optimization will greatly simplify the linear system. This modification is implemented by making trivial modifications to System 2 .

Our second modification to the above scheme is more important: we want to optimize more than the max-stretch. The first possibility would be to use in an online framework our offline heuristic for the Pareto minimization of max-stretch. To do so, instead of using a binary search and System 2 to compute the best achievable max-stretch, we use Algorithm 3 where, at Step 4, we compute the best achievable max-stretch rather than the optimal one. This way we define our OnLINE-PARETo heuristics.

Another possible approach would be to specify that each job should be scheduled in a manner that minimizes its own stretch value, while maintaining the overall maximal stretch value obtained. For example, one could theoretically try to minimize the sum-stretch under the condition that the maxstretch be optimal. However, as we have seen, minimizing the sum-stretch is an open problem. So we consider a heuristic approach expressed by System (6).

$$
\begin{aligned}
& \text { Minimize } \sum_{j=1}^{n} w_{j}\left(\left(\sum_{t}\left(\sum_{i=1}^{m} \alpha_{i, j}^{(t)}\right) \frac{\sup I_{t}\left(\mathcal{S}^{*}\right)+\inf I_{t}\left(\mathcal{S}^{*}\right)}{2}\right)-r_{j}\right) \\
& \text { Under the CONSTRAINTS } \\
& \begin{cases}(6 \mathrm{a}) \quad \forall i, \forall j, \forall t, \quad r_{j} \geqslant \sup I_{t}\left(\mathcal{S}^{*}\right) \Rightarrow \alpha_{i, j}^{(t)}=0 \\
(6 \mathrm{~b}) \quad \forall i, \forall j, \forall t, \quad d_{j}\left(\mathcal{S}^{*}\right) \leqslant \inf I_{t}\left(\mathcal{S}^{*}\right) \Rightarrow \alpha_{i, j}^{(t)}=0 \\
(6 \mathrm{c}) \quad \forall t, \forall i, \quad \sum_{j} \alpha_{i, j}^{(t)} \cdot p_{i, j} \leqslant \sup I_{t}\left(\mathcal{S}^{*}\right)-\inf I_{t}\left(\mathcal{S}^{*}\right) \\
(6 \mathrm{~d}) \quad \forall j, \quad \sum_{t} \sum_{i} \alpha_{i, j}^{(t)}=1\end{cases}
\end{aligned}
$$

This system ensures that each job is completed no later than the deadline defined by the optimal (offline) max-stretch $\mathcal{S}^{*}$. Then, under this constraint, this system attempts to minimize an objective that resembles a rational relaxation of the sum-stretch (or more generally of the sum weighted flow) using as an approximation of the completion time, the weighted sum of the average execution times of a job. As we do not know the precise time within an interval when a part of a job will be scheduled, we approximate it by the mean time of the interval. (This heuristic obviously offers no guarantee on the sum-stretch achieved.) This way, we obtain the following online algorithm. Each time a new job arrives: 
1. Preempt the running job (if any).

2. Compute the best achievable max-stretch $\mathcal{S}^{*}$, considering the decisions already made.

3. With the deadlines and intervals defined by the max-stretch $\mathcal{S}^{*}$, solve System (6).

At this point, we define three variants to produce the schedule. The first, which we call OnLINE, assigns work simply using the values found by the linear program for the $\alpha$ variables:

4. For a given processor $P_{i}$, and a given interval $I_{t}\left(\mathcal{S}^{*}\right)$, all jobs $J_{j}$ that complete their fraction on that processor during the same interval (i.e., all jobs $J_{j}$ such that $\sum_{t^{\prime}>t} \alpha_{i, j}^{\left(t^{\prime}\right)}=0$ ) are scheduled under the SWRPT policy in that interval. We call these jobs terminal jobs (for $P_{i}$ and $I_{t}\left(\mathcal{S}^{*}\right)$ ). The non-terminal jobs scheduled on $P_{i}$ during interval $I_{t}\left(\mathcal{S}^{*}\right)$ are only executed in $I_{t}\left(\mathcal{S}^{*}\right)$ after all terminal jobs have finished.

The second variant we consider, ONLINE-EDF, attempts to make changes to the schedule at the processor level to improve the overall max- and sum-stretch attained:

4. Consider a processor $P_{i}$. The fractions $\alpha_{i, j}$ of the jobs that must be partially executed on $P_{i}$ are processed on $P_{i}$ under a list scheduling policy based on the following order: the jobs are ordered according to the interval in which their share is completed (according to the solution of the linear program), with ties being broken by the SWRPT policy.

Finally, we propose a third variant, ONLINE-EGDF, that creates a global priority list:

4. The (active) jobs are processed under a list scheduling policy, using the strategy outlined in Section 2.3 to deal with restricted availabilities. Here, the jobs are totally ordered by the interval in which their total work is completed, with ties being broken by the SWRPT policy.

The validity of these heuristic approaches will be assessed through simulations in the section 9 .

\section{Simulations}

To evaluate the efficacy of various scheduling strategies when optimizing stretch-based metrics, we implemented a simulator using the SimGrid toolkit [24], based on the biological sequence comparison scenario. The application and platform models used in the resulting simulator are derived from our initial observations of the GriPPS system, described in Section 2. Our primary goal is to evaluate the proposed heuristics in realistic conditions that include partial replication of target sequence databases across the available computing resources. The remainder of this section outlines the experimental variables we considered and presents results describing the behavior of the heuristics in question under various parametrizations of the platform and application models.

\subsection{Simulation Settings}

The platform and application models that we address in this work are quite flexible, resulting in innumerable variations in the range of potentially interesting combinations. To facilitate our studies, we concretely define certain features of the system that we believe to be useful in describing realistic execution scenarios. We consider in particular six such features.

Platform size: Typically, a given biological database such as those considered in this work, would be replicated at various sites, at which comparisons against this database may be performed. Generally, the number of sites in a simulated system provides a basic measure of the aggregate power of the platform. This parameter specifies the exact number of sites in the simulated platform. Without loss of generality, we arbitrarily define each site to contain 10 processors.

Processor power: Our model assumes that all the processors at any given site are equivalent, and each processor is assumed to have access to all databases located there. Thus for each site, a single processor value represents the processing power at that site. We choose processor power values using benchmark results from our previous work.

Number of databases: Applications such as GriPPS can accommodate multiple reference databases. Our model allows for any number of distinct databases to exist throughout the system. 
Database size: Our previous work demonstrated that the processing time needed to service a user request targeting a particular database varies linearly according to the number of sequences found in the database in question. We choose such values from a continuous range of realistic database sizes, with the job size for jobs targeting a particular database scaled accordingly.

Database availability: A particular database may be replicated at multiple sites, and a single site may host copies of multiple databases. We account for these two eventualities by associating with each database a probability of existence at each site. The same database availability applies to all databases in the system. We further ensure that each database is available at at least one site, and each site hosts at least one database.

Workload density: For a particular database, we define the workload density of a system to be the ratio, on average, of the aggregate job size of user requests against that database to the aggregate computational power available to serve such requests. Workload density expresses a notion of the "load" of the system. This parameter, along with the size of the database, define the frequency of job arrivals in the system.

We define a simulation configuration as a set of specific values for each of these six properties. Once defined, concrete simulation instances are constructed by realizing random series for any random variables in the system. In particular, two models are created for each instance: a platform model and a workload model. The former is specified first by defining the appropriate number of 10-node sites and assigning corresponding processor power values. Next, a size is assigned to each database, and it is replicated according to the simulation's database availability parameter. Finally, the workload model is realized by first generating a series of jobs for each database, using a Poisson process for job inter-arrival times, with a mean that is computed to attain the desired workload density. The database-specific workloads are then merged and sorted to obtain the final workload. Jobs may arrive between the time at which the simulation starts and 15 minutes thereafter.

In this simulation study, we use empirical values observed in the GriPPS system logs to define a realistic range of database sizes and to generate appropriate values for processor speeds. The remaining four parameters - platform size, number of distinct databases, database availability, and workload density are the simulation values that vary in our study. We discuss further the specifics of the experimental design and our simulation results in Section 9.3.

\subsection{Optimization of the Online Heuristic}

To motivate the variants of our online heuristic described in Section 8, we conduct a series of experiments to evaluate their effect. In particular, we consider a non-optimized version of the online heuristic, which stops after Step 2. We consider job workloads of average density varying between 0.0125 to 4.00 , over a range of average job lengths between 15 and 60 seconds. For each job size/workload density combination evaluated, we simulate the execution of 5000 instances, recording the maximum and sum stretch of jobs in the workload achieved with both the optimized and non-optimized versions of the online heuristic. The max-stretch of each is then divided by the max-stretch achieved by the optimal algorithm, yielding a degradation factor for both heuristics on that run. Since the optimal sum-stretch is not known, we observe the sum-stretch of the optimized online heuristic relative to the non-optimized version. Figures 5(a) and 5(b) present the max-stretch and sum-stretch results, respectively. In the first plot, the average maxstretch degradation, compared to the optimal result, for both versions of the heuristic over the 5000 runs of a given configuration is plotted against the workload density of that configuration. The second plot depicts the gain for the sum-stretch metric for the optimized heuristic, relative to the non-optimized version. These results strongly motivate the use of the optimizations encoded by the linear program depicted in System (6).

\subsection{Simulation Results and Analysis}

We have implemented in our simulator a number of scheduling heuristics that we plan to compare. First, we have implemented OfFLINE, corresponding to the algorithm described in Section 6 that solves the optimal max-stretch problem. Three versions of the online heuristic are also implemented, designated as OnLINE, ONLINE-EDF, and OnLINE-EGDF. Next, we consider the SWRPT, SRPT, and SPT heuristics discussed in Section 5. Then, we consider the two online heuristics proposed by Bender et al. that were briefly described in Section 8.2. We also include two greedy strategies. First, MCT ("minimum 


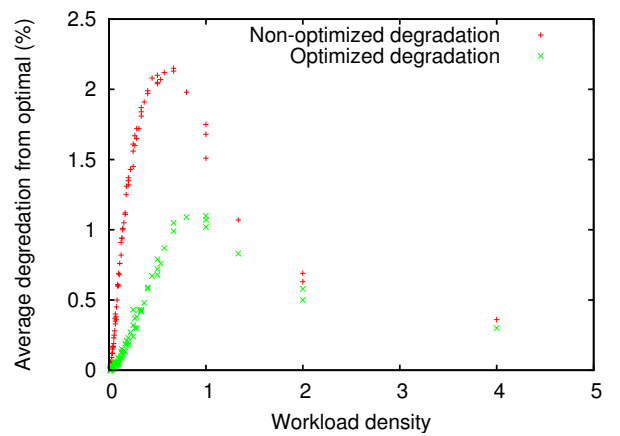

(a) Max-stretch degradation from optimal of both versions of the online heuristic

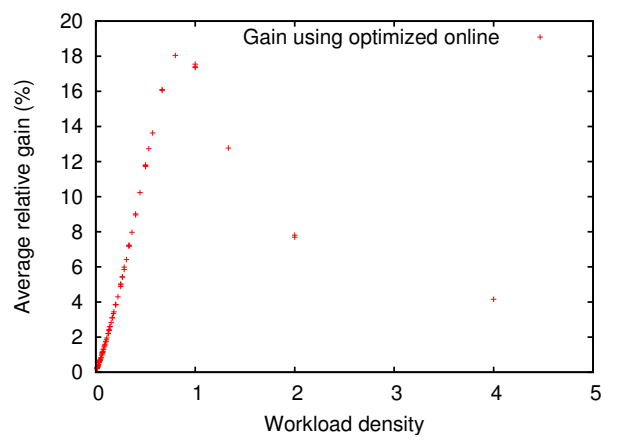

(b) Sum-stretch gain of the optimized version, relative to the non-optimized version

Figure 5: Comparison of the optimized and non-optimized versions of the online heuristic.

completion time") simply schedules each job as it arrives on the processor that would offer the best job completion time. The FCFS-DIV heuristic extends this approach to take advantage of the fact that jobs are divisible, by employing all resources that are able to execute the job (using the strategy laid out in Section 2.3). Note that neither MCT nor FCFS-DIV makes any changes to work that has already been scheduled. Finally, as a basic reference, we consider a list-scheduling heuristic with random job order denoted RAND. This heuristic works as follows: initially, we randomly build an order on the jobs that may arrive; then RAND list-schedules the jobs while using this list to define priorities, and while using the divisibility property. All the single processor heuristics (SWRPT, SRPT, SPT, and Bender et al.'s) are extended to the multi-processor case using the Algorithm 1 previously described in Section 2.3.

As mentioned earlier, two of the six parameters of our model reflect empirical values determined in our previous work with the GriPPS system [26]. Processor speeds are chosen randomly from one of the six reference platforms we studied, and we let database sizes vary continuously over a range of 10 megabytes to 1 gigabyte, corresponding roughly to GriPPS database sizes. Thus, our experimental results examine the behaviors of the aforementioned heuristics as we vary our four experimental parameters:

platforms of 3,10 , and 20 clusters (sites) with 10 processors each;

applications with 3, 10, and 20 distinct databases;

database availabilities of $30 \%, 60 \%$, and $90 \%$ for each database; and

workload density factors of $0.75,1.0,1.25,1.5,2.0$, and 3.0 .

The resulting experimental framework has 162 configurations. For each configuration, 200 platforms and application instances are randomly generated and the simulation results for each of the studied heuristics is recorded. Table 1 presents the aggregate results from these simulations; finer-grained results based on various partitionings of the data may be found in extended version of this document [28].

Above all, we note that the MCT heuristic - effectively the policy in the current GriPPS system - is unquestionably inappropriate for max-stretch optimization: MCT was over 38 times worse on average than the best heuristic. Its deficiency might arguably be tolerable on small platforms but, in fact, MCT yielded max-stretch performance over 16 times worse than the best heuristic in all simulation configurations. Even after addressing the primary limitation that the divisibility property is not utilized, the results are still disappointing: FCFS-DIV is on average 5.1 times worse in terms of max-stretch than the best approach we found. One of the principal failings of the MCT and FCFS-Div heuristics is that they are non-preemptive. By forcing a small task that arrives in a heavily loaded system to wait, non-preemptive schedulers cause such a task to be inordinately stretched relative to large tasks that are already running.

Experimentally, we find that the first two of the three online heuristics we propose are consistently near-optimal (within 4\%o on average) for max-stretch optimization. The third heuristic, OnLINE-EGDF, actually achieves consistently good sum-stretch (within $3 \%$ of the best observed sum-stretch), but at the expense of its performance for the max-stretch metric (within $4 \%$ of the optimal). This is not entirely surprising as this heuristic ignores a significant portion of the fine-tuned schedule generated by

\footnotetext{
${ }^{6}$ BENDER98 results are limited to 3-cluster platforms, due to prohibitive overhead costs (discussed in Section 9.3).
} 


\begin{tabular}{r|rrr||rrr} 
& \multicolumn{3}{|c||}{ Max-stretch } & \multicolumn{3}{c}{ Sum-stretch } \\
& Mean & SD & Max & Mean & SD & Max \\
\hline OFFLINE & 1.0000 & 0.0000 & 1.0000 & 1.4051 & 0.2784 & 2.6685 \\
OFFLINEPARETO & 1.0000 & 0.0000 & 1.0000 & 1.2986 & 0.2605 & 3.5090 \\
ONLINE & 1.0039 & 0.0145 & 1.2420 & 1.0458 & 0.0439 & 1.5069 \\
ONLINE-EDF & 1.0040 & 0.0156 & 1.6886 & 1.0450 & 0.0432 & 1.5016 \\
ONLINE-EGDF & 1.0331 & 0.0622 & 1.6613 & 1.0024 & 0.0052 & 1.1095 \\
SWRPT & 1.0386 & 0.0729 & 2.0566 & 1.0003 & 0.0014 & 1.0384 \\
SRPT & 1.0596 & 0.1027 & 2.1012 & 1.0048 & 0.0074 & 1.1179 \\
SPT & 1.0576 & 0.1032 & 2.1297 & 1.0020 & 0.0048 & 1.1263 \\
BENDER98 & 1.0415 & 0.0971 & 2.1521 & 1.0028 & 0.0075 & 1.1393 \\
BENDER02 & 2.9859 & 2.7071 & 23.5446 & 1.2049 & 0.3087 & 6.6820 \\
FCFS-DIV & 5.1353 & 6.6792 & 65.9073 & 1.3767 & 0.7224 & 15.4213 \\
MCT & 38.4276 & 24.2626 & 156.3778 & 51.9606 & 36.5202 & 154.1519 \\
RAND & 4.6568 & 6.9107 & 87.9141 & 1.2355 & 0.4827 & 10.8549
\end{tabular}

Table 1: Aggregate statistics over all 162 platform/application configurations.

the linear program designed to optimize the max-stretch. Furthermore, our three online heuristics have far better sum-stretch than the OfFLINEPARETO (which is on average almost 30\% away of the best observed sum-stretch). This result validates our heuristic optimization of sum-stretch as expressed by Linear Program 6. As forecasted, OfFLINEPARETO has a significantly better average sum-stretch than OFFLINE.

We also observe that SWRPT, SRPT, and SPT are all quite effective at sum-stretch optimization. Each is on average within $5 \%$ of the best observed sum-stretch for all configurations. In particular, SWRPT produces a sum-stretch that is on average $0.3 \%$ within the best observed sum-stretch, and attaining a sum-stretch within $4 \%$ of the best sum-stretch in all of the roughly 32,000 instances. However, it should be noted that these heuristics may lead to starvation. Jobs may be delayed for an arbitrarily long time, particularly when a long series of small jobs is submitted sequentially (the $(n+1)^{\text {th }}$ job being released right after the termination of the $n^{\text {th }}$ job). Our analysis of the GriPPS application logs has revealed that such situations occur fairly often due to automated processes that submit jobs at regular intervals. By optimizing max-stretch in lieu of sum-stretch, the possibility of starvation is eliminated.

Next, we find that the Bender98 and Bender02 heuristics are not practically useful in our scheduling context. The results shown in Table 1 for the BENDER98 heuristic comprise only 3-cluster platforms; simulations on larger platforms were practically infeasible, due to the algorithm's prohibitive overhead costs. Effectively, for an $n$-task workload, the BENDER98 heuristic solves $n$ optimal max-stretch problems, many of which are computationally equivalent to the full $n$-task optimal solution. In several cases the desired workload density required thousands of tasks, rendering the BENDER98 algorithm intractable. To roughly compare the overhead costs of the various heuristics, we ran a small series of simulations using only 3 -cluster platforms. The results of these tests indicate that the scheduling time for a 15-minute workload was on average under $0.28 \mathrm{~s}$ for any of our online heuristics, and $0.54 \mathrm{~s}$ for the offline optimal algorithm (with $0.35 \mathrm{~s}$ spent in the resolution of the linear program and $0.19 \mathrm{~s}$ spent in the online phases of the scheduler); by contrast, the average time spent in the BENDER98 scheduler was $19.76 \mathrm{~s}$. The scheduling overhead of BENDER02 is far less costly (on average $0.23 \mathrm{~s}$ of scheduling time in our overhead experiments), but in realistic scenarios for our application domain, the competitive ratios it guarantees are ineffective compared with our online heuristics for max-stretch optimization. Note that the bad performance of BENDER02 is not due to the way we adapt single-machine algorithms to unrelated machines configurations (see Section 2.3). Indeed, similar observations can be done when restricting to single-machine configurations (see Table 2).

Finally, we remark that the RAND heuristic is slightly better than the FCFS-DIV for both metrics. Moreover, RAND is only $24 \%$ away from the best observed sum-stretch on average. This leads us to think that the sum-stretch may not be a discriminating objective for our problem. Indeed, it looks as if, whatever the policy, any list-scheduling heuristic delivers good performance for this metric. 


\begin{tabular}{r|rrr||rrr} 
& \multicolumn{3}{|c||}{ Max-stretch } & \multicolumn{3}{c}{ Sum-stretch } \\
& Mean & SD & Max & Mean & SD & Max \\
\hline OFFLINE & 1.0000 & 0.0000 & 1.0000 & 1.0413 & 0.0593 & 1.6735 \\
ONLINE & 1.0016 & 0.0149 & 1.6344 & 1.0549 & 0.0893 & 1.8134 \\
SWRPT & 1.1316 & 0.2071 & 3.1643 & 1.0001 & 0.0009 & 1.0398 \\
SRPT & 1.1242 & 0.2003 & 3.0753 & 1.0139 & 0.0212 & 1.2576 \\
SPT & 1.1961 & 0.2667 & 3.9752 & 1.0229 & 0.0296 & 1.3573 \\
BENDER98 & 1.1200 & 0.1766 & 2.5428 & 1.0194 & 0.0279 & 1.4466 \\
BENDER02 & 3.5422 & 2.4870 & 21.4819 & 2.9872 & 1.9599 & 15.0019 \\
FCFS-DIV & 8.7762 & 9.1900 & 80.7465 & 6.8979 & 7.7409 & 88.2449 \\
RAND & 11.3059 & 11.1981 & 125.3726 & 5.8227 & 6.3942 & 68.0009
\end{tabular}

Table 2: Aggregate statistics for a single machine for all application configurations.

\section{Conclusion}

Our initial goal was to minimize the maximum stretch. We have presented a polynomial-time algorithm to solve this problem offline. We have also proposed some heuristics to solve this problem online. Through simulations we have shown that these heuristics are far more efficient than the pre-existing guaranteed heuristics, and do not have the risk of job starvation present in classical simple scheduling heuristics like shortest remaining processing time. Along the way we have established some NP-completeness and competitiveness results. Table 3 summarizes the main complexity results presented in this document as well as related work. Minimizing $\max w_{j} F_{j}$ is polynomial as soon as divisibility or preemption is allowed whereas $\sum w_{j} F_{j}$ is always strongly NP-hard. $\sum F_{j}$ is easy only on simple settings (one processor with preemption of related processors with divisibility) and is strongly NP-hard in all other settings. The main problem whose complexity is still open is $\left\langle 1\left|r_{j}, p m t n\right| \sum S_{j}\right\rangle$ even if (as we already have mentioned in Section 5.1) Polynomial Time Approximation Scheme (PTAS) have been proposed for this problem. Some other questions remain open, like:

\begin{tabular}{|c|c|c|c|}
\cline { 2 - 4 } \multicolumn{1}{c|}{} & $\beta=\emptyset$ & $\beta=p m t n$ & $\beta=$ div \\
\hline$\left\langle 1\left|r_{j} ; \beta\right| \max w_{j} F_{j}\right\rangle$ & $N P([5])$ & $\downarrow$ & $\downarrow$ \\
\hline$\left\langle P\left|r_{j} ; \beta\right| \max w_{j} F_{j}\right\rangle$ & $\uparrow$ & $\downarrow$ & $\downarrow$ \\
\hline$\left\langle Q\left|r_{j} ; \beta\right| \max w_{j} F_{j}\right\rangle$ & $\uparrow$ & $\downarrow$ & $\downarrow$ \\
\hline$\left\langle R\left|r_{j} ; \beta\right| \max w_{j} F_{j}\right\rangle$ & $\uparrow$ & $P$ (Lin. Prog. Sec. 6.2) & $P$ (Lin. Prog. Sec. 6.1) \\
\hline \hline$\left\langle 1\left|r_{j} ; \beta\right| \sum F_{j}\right\rangle$ & $N P([29])$ & $P$ (SRPT [1]) & $\downarrow$ \\
\hline$\left\langle P\left|r_{j} ; \beta\right| \sum F_{j}\right\rangle$ & $\uparrow$ & $N P($ Numerical-3DM [3]) & $\downarrow$ \\
\hline$\left\langle Q\left|r_{j} ; \beta\right| \sum F_{j}\right\rangle$ & $\uparrow$ & $\uparrow$ & $P$ (SRPT + Sec. 2.3) \\
\hline$\left\langle R\left|r_{j} ; \beta\right| \sum F_{j}\right\rangle$ & $\uparrow$ & $\uparrow$ & $N P(3 \mathrm{DM}$, Sec. 4) \\
\hline \hline$\left\langle 1\left|r_{j} ; \beta\right| \sum S_{j}\right\rangle$ & $N P($ Sec. 5.1) & $?$ & $?$ \\
\hline$\left\langle P\left|r_{j} ; \beta\right| \sum S_{j}\right\rangle$ & $\uparrow$ & $?$ & $?$ \\
\hline$\left\langle Q\left|r_{j} ; \beta\right| \sum S_{j}\right\rangle$ & $\uparrow$ & $?$ & $?$ \\
\hline$\left\langle R\left|r_{j} ; \beta\right| \sum S_{j}\right\rangle$ & $\uparrow$ & $?$ & ? \\
\hline \hline$\left\langle 1\left|r_{j} ; \beta\right| \sum w_{j} F_{j}\right\rangle$ & $N P([29])$ & $N P($ Numerical-3DM [22]) & $\uparrow$ \\
\hline$\left\langle P\left|r_{j} ; \beta\right| \sum w_{j} F_{j}\right\rangle$ & $\uparrow$ & $\uparrow$ & $\uparrow$ \\
\hline$\left\langle Q\left|r_{j} ; \beta\right| \sum w_{j} F_{j}\right\rangle$ & $\uparrow$ & $\uparrow$ & $\uparrow$ \\
\hline$\left\langle R\left|r_{j} ; \beta\right| \sum w_{j} F_{j}\right\rangle$ & $\uparrow$ & $\uparrow$ & $\uparrow .1)$ \\
\hline
\end{tabular}

Table 3: Summary of complexity results.

- What is the complexity of $\langle R|$ div; $r_{j} \mid$ Pareto $\left.S_{\max }\right\rangle$, the Pareto minimization of max-stretch on unrelated machines under the divisible load model

- Are there some approximation algorithms minimizing the sum-stretch on unrelated machines under the divisible load model? 
- Are there some algorithms with a better competitivity factor than 2 for the minimization of sumstretch on a single processor?

- Processor Sharing is a scheduling policy where time units are divided arbitrarily finely between jobs and where all jobs currently in the system get an equal share of the machine. In [5, 4], Bender et al. claim that Processor Sharing has a competitive ratio of $\Omega(n)$ for max-stretch where $n$ is the number of jobs. This is thus very bad compared to the known $O(\sqrt{\Delta})$ competitive algorithms. However in the instance they use, $\Delta$ grows with $n$ (more precisely $\Delta=2^{n}$ ). Therefore, Processor Sharing may not be such a bad algorithm for the max-stretch minimization. It is not hard (at least numerically) to see that the competitive ratio is $\Omega(\sqrt{\Delta})$. The open question is therefore: what is the competitive ratio of Processor Sharing for max-stretch ?

Beside all the theoretical considerations, we think that the study presented in this article clearly demonstrates the superiority of our algorithms in terms of fairness and efficiency compared to currently implemented scheduling algorithm in the GriPPS application. In particular, we hope that this study has shown the major importance of divisibility and preemption in this framework and that such techniques will soon be used in practice.

\section{Acknowledgments}

We would like to thank Lionel Eyraud for the many insightful discussions we had together. We also like to thank Michael Bender who presented us some known algorithms for minimizing max-stretch on one machine with preemption. Last, we would like to thank the reviewers for their thorough reading and insightful comments.

\section{References}

[1] K.R. Baker. Introduction to Sequencing and Scheduling. Wiley, New York, 1974.

[2] K.R. Baker, E.L. Lawler, J.K. Lenstra, and A.H.G Rinnooy Kan. Preemptive scheduling of a single machine to minimize maximum cost subject to release dates and precedence constraints. Operations Research, 31(2):381-386, March 1983.

[3] Philippe Baptiste, Peter Brucker, Marek Chrobak, Christoph Dürr, Svetlana A. Kravchenko, and Francis Sourd. The complexity of mean flow time scheduling problems with release times. Journal of Scheduling, 10(2):139-146, April 2007.

[4] Michael A. Bender. New Algorithms and Metrics for Scheduling. PhD thesis, Harvard University, May 1998.

[5] Michael A. Bender, Soumen Chakrabarti, and S. Muthukrishnan. Flow and stretch metrics for scheduling continuous job streams. In Proceedings of the 9th Annual ACM-SIAM Symposium On Discrete Algorithms (SODA'98), pages 270-279. Society for Industrial and Applied Mathematics, 1998.

[6] Michael A. Bender, S. Muthukrishnan, and Rajmohan Rajaraman. Improved algorithms for stretch scheduling. In SODA '02: Proceedings of the thirteenth annual ACM-SIAM symposium on Discrete algorithms, pages 762-771, Philadelphia, PA, USA, 2002. Society for Industrial and Applied Mathematics.

[7] Michael A. Bender, S. Muthukrishnan, and Rajmohan Rajaraman. Approximation algorithms for average stretch scheduling. J. of Scheduling, 7(3):195-222, 2004.

[8] D. Bertsekas and R. Gallager. Data Networks. Prentice Hall, 1987.

[9] V. Bharadwaj, D. Ghose, V. Mani, and T.G. Robertazzi. Scheduling Divisible Loads in Parallel and Distributed Systems. IEEE Computer Society Press, 1996. 
[10] Christophe Blanchet, Christophe Combet, Christophe Geourjon, and Gilbert Deléage. MPSA: Integrated System for Multiple Protein Sequence Analysis with client/server capabilities. Bioinformatics, 16(3):286-287, 2000.

[11] J. Blazewicz, K.H. Ecker, E. Pesch, G. Schmidt, and J. Weglarz. Handbook on Scheduling: From Theory to Applications. International Handbooks on Information Systems. Springer, 2007. ISBN: 978-3-540-28046-0.

[12] Jacek Blazewicz. Scheduling dependent tasks with different arrival times to meet deadlines. In Heinz Beilner and Erol Gelenbe, editors, Modelling and Performance Evaluation of Computer Systems: Proceedings of the International Workshop, pages 57-65. North-Holland, 1976.

[13] R. C. Braun, Kevin T. Pedretti, Thomas L. Casavant, Todd E. Scheetz, C. L. Birkett, and Chad A. Roberts. Parallelization of local BLAST service on workstation clusters. Future Generation Computer Systems, 17(6):745-754, 2001.

[14] Chandra Chekuri and Sanjeev Khanna. Approximation schemes for preemptive weighted flow time. In Proceedings of the thiry-fourth annual ACM symposium on Theory of computing, pages 297-305. ACM Press, 2002.

[15] Aaron E. Darling, Lucas Carey, and Wu chun Feng. The Design, Implementation, and Evaluation of mpiBLAST. In Proceedings of ClusterWorld 2003, 2003.

[16] M. L. Dertouzos. Control robotics: the procedural control of physical processes. In Proceedings of IFIP Congress, pages 897-813, 1974.

[17] M. Protasi G. Ausiello, A. D'Atri. Structure preserving reductions among convex optimization problems. Journal of Computer and System Sciences, 21(1):136-153, August 1980.

[18] M. R. Garey and D. S. Johnson. Computers and Intractability, a Guide to the Theory of NPCompleteness. W. H. Freeman and Company, 1991.

[19] Teofilo Gonzalez and Sartaj Sahni. Open shop scheduling to minimize finish time. J. ACM, 23(4):665-679, 1976.

[20] GriPPS webpage at http://gripps.ibcp.fr/, 2005.

[21] D. S. Johnson. Approximation algorithms for combinatorial problems. Journal of Computer and System Sciences, 9:256-278, 1974.

[22] Jacques Labetoulle, Eugene L. Lawler, J.K. Lenstra, and A.H.G. Rinnooy Kan. Preemptive scheduling of uniform machines subject to release dates. In W. R. Pulleyblank, editor, Progress in Combinatorial Optimization, pages 245-261. Academic Press, 1984.

[23] Eugene L. Lawler and Jacques Labetoulle. On preemptive scheduling of unrelated parallel processors by linear programming. Journal of the Association for Computing Machinery, 25(4):612-619, 1978.

[24] Arnaud Legrand, Loris Marchal, and Henri Casanova. Scheduling Distributed Applications: The SimGrid Simulation Framework. In Proceedings of the 3rd IEEE Symposium on Cluster Computing and the Grid, 2003.

[25] Arnaud Legrand, Alan Su, and Frédéric Vivien. Off-line scheduling of divisible requests on an heterogeneous collection of databanks. Research report 5386, INRIA, November 2004. Also available as LIP, ENS Lyon, research report 2004-51.

[26] Arnaud Legrand, Alan Su, and Frédéric Vivien. Off-line scheduling of divisible requests on an heterogeneous collection of databanks. In Proceedings of the 14th Heterogeneous Computing Workshop, Denver, Colorado, USA, April 2005. IEEE Computer Society Press.

[27] Arnaud Legrand, Alan Su, and Frederic Vivien. Minimizing the stretch when scheduling flows of biological requests. In Symposium on Parallelism in Algorithms and Architectures SPAA'2006. ACM Press, 2006. 
[28] Arnaud Legrand, Alan Su, and Frédéric Vivien. Minimizing the stretch when scheduling flows of divisible requests. Research Report RR2008-08, LIP, École Normale Supérieure de Lyon, February 2008. This is a revised version of the LIP research report RR2006-19. Also available as INRIA research report 6002 http://hal.inria.fr/inria-00108524.

[29] J.K. Lenstra, A.H.G. Rinnooy Kan, and P. Brucker. Complexity of machine scheduling problems. Annals of Discrete Mathematics, 1:343-362, 1977.

[30] Laurent Massoulié and James Roberts. Bandwidth sharing: Objectives and algorithms. Transactions on Networking, 10(3):320-328, june 2002.

[31] Nicole Megow. Performance analysis of on-line algorithms in machine scheduling. Diplomarbeit, Technische Universität Berlin, April 2002.

[32] Perry L. Miller, Prakash M. Nadkarni, and N. M. Carriero. Parallel computation and FASTA: confronting the problem of parallel database search for a fast sequence comparison algorithm. Computer Applications in the Biosciences, 7(1):71-78, 1991.

[33] S. Muthukrishnan, Rajmohan Rajaraman, Anthony Shaheen, and Johannes Gehrke. Online scheduling to minimize average stretch. In IEEE Symposium on Foundations of Computer Science, pages 433-442, 1999.

[34] Andreas S. Schulz and Martin Skutella. The power of $\alpha$-points in preemptive single machine scheduling. Journal of Scheduling, 5(2):121-133, 2002. DOI:10.1002/jos.093.

[35] René Sitters. Complexity of preemptive minsum scheduling on unrelated parallel machines. Journal of Algorithms, 57(1):37-48, September 2005.

[36] Petr Slavík. A tight analysis of the greedy algorithm for set cover. In STOC '96: Proceedings of the twenty-eighth annual ACM symposium on Theory of computing, pages 435-441, New York, NY, USA, 1996. ACM Press.

[37] Wayne E. Smith. Various optimizers for single-stage production. Naval Research Logistics Quarterly, $3: 59-66,1956$ 